\title{
Forecasting propagation and evolution of CMEs in an operational setting: What has been learned
}

Yihua Zheng, ${ }^{1}$ Peter Macneice, ${ }^{1}$ Dusan Odstrcil, ${ }^{1,2}$ M. L. Mays, ${ }^{1,3}$ Lutz Rastaetter, ${ }^{1}$ Antti Pulkkinen, ${ }^{1}$ Aleksandre Taktakishvili, ${ }^{1,3}$ Michael Hesse, ${ }^{1}$ M. Masha Kuznetsova, ${ }^{1}$ Hyesook Lee, ${ }^{4}$ and Anna Chulaki ${ }^{1,5}$

Received 28 January 2013; revised 21 August 2013; accepted 5 September 2013; published 2 October 2013.

[1] One of the major types of solar eruption, coronal mass ejections (CMEs) not only impact space weather, but also can have significant societal consequences. CMEs cause intense geomagnetic storms and drive fast mode shocks that accelerate charged particles, potentially resulting in enhanced radiation levels both in ions and electrons. Human and technological assets in space can be endangered as a result. CMEs are also the major contributor to generating large amplitude Geomagnetically Induced Currents (GICs), which are a source of concern for power grid safety. Due to their space weather significance, forecasting the evolution and impacts of CMEs has become a much desired capability for space weather operations worldwide. Based on our operational experience at Space Weather Research Center at NASA Goddard Space Flight Center (http://swrc.gsfc.nasa.gov), we present here some of the insights gained about accurately predicting CME impacts, particularly in relation to space weather operations. These include: 1. The need to maximize information to get an accurate handle of three-dimensional (3-D) CME kinetic parameters and therefore improve CME forecast; 2 . The potential use of CME simulation results for qualitative prediction of regions of space where solar energetic particles (SEPs) may be found; 3 . The need to include all CMEs occurring within a $\sim 24 \mathrm{~h}$ period for a better representation of the CME interactions; 4. Various other important parameters in forecasting CME evolution in interplanetary space, with special emphasis on the CME propagation direction. It is noted that a future direction for our CME forecasting is to employ the ensemble modeling approach.

Citation: Zheng, Y., et al. (2013), Forecasting propagation and evolution of CMEs in an operational setting: What has been learned, Space Weather, 11, 557-574, doi:10.1002/swe.20096.

\section{Introduction}

[2] The three main types of solar transient/structure responsible for space weather are: 1 . Solar flares, 2. Coronal mass ejections (CMEs), and 3. Corotating interaction regions (CIRs) and the accompanying high-speed solar wind streams.

[3] Solar flares and CMEs are arguably the two most energetic/catastrophic solar phenomena [e.g., Hudson, 2011;

\footnotetext{
${ }^{1}$ NASA Goddard Space Flight Center, Space Weather Laboratory, Code 674.0, Greenbelt, Maryland, USA.

${ }^{2}$ George Mason University, Fairfax, Virginia, USA.

${ }^{3}$ Catholic University of America, NASA/GSFC, Greenbelt, Maryland, USA.

${ }^{4}$ Korean Meteorological Administration, South Korea.

${ }^{5}$ Sigma Space Corp., Lanham, Maryland, USA.

Corresponding author: Y. Zheng, NASA Goddard Space Flight Center, Space Weather Laboratory, Code 674.0, Greenbelt, MD 20771, USA. (Yihua.Zheng@nasa.gov)

○2013. American Geophysical Union. All Rights Reserved. 1542-7390/13/10.1002/swe.20096
}

Webb and Howard, 2012; Qiu et al., 2004; Qiu and Yurchyshyn, 2005; Yashiro et al., 2005; Wang and Zhang, 2007; Zhang et al., 2001 and references therein]. A CME is the spectacular ejection of mass and magnetic field outward from the solar atmosphere into interplanetary space. Although it is often associated with a solar flare, a one-to-one correlation is not always observed. Interplanetary CMEs (ICMEs) are the interplanetary manifestations of CMEs typically remote sensed by coronagraphs. The speed of CMEs ranges from $\sim 200 \mathrm{~km} / \mathrm{s}$ to $\sim 3000 \mathrm{~km} / \mathrm{s}$, with the mass often totaling more than $10^{12} \mathrm{~kg}$ and the kinetic energy exceeding $10^{15} \mathrm{~J}$. Often flare (electromagnetic) radiation energy can be comparable to CME kinetic energy. The relationship between a flare and its associated CME has been interpreted in various ways. Some studies suggest that rather than being distinct physical processes, they could be merely different manifestations of the same physical process [e.g., Zhang et al., 2001; 2004].

[4] In comparison with solar flares and CIRs, CMEs are more likely to have significant (at times severe) space weather impacts. Earth-directed CMEs (often originating from the disk center - within a longitude range of $\pm 30^{\circ}$ ) 
are the drivers of major geomagnetic storms [e.g., Cane and Richardson, 2003; Gopalswamy et al., 20005a, 2005b; Gopalswamy, 2006], capable of causing rapid and large magnetic field disturbances, enhanced currents in space and on the ground (the so-called Geomagnetically Induced Currents - GICs), and enhanced radiation in our near-Earth environment - both from energetic electrons and from ions. CMEs pose serious threats to spacecraft components and operations, communication and navigation systems, astronauts either in extravehicular activity or behind a protective shielding [Lanzerotti, 2004], as well as ground systems such as power grids and pipelines [e.g., Boteler et al., 1998; Pirjola, 2007].

[5] Another aspect of CMEs' importance in space weather lies in their roles in accelerating solar energetic particles (SEPs). Large SEP events ("large" means high particle flux for protons (or other ion species) at energies equal or above $10 \mathrm{MeV}$ here) constitute a serious radiation hazard to spacecraft systems and humans in space.

[6] Physical understanding of SEPs has evolved significantly over the years. Originally, solar flares were believed to be the source of the energetic particles; such a view largely reflected a lack of CME observations. A paradigm shift with regard to SEPs occurred around the 1980s and 1990s [e.g., Reames, 1999]. Attention was now drawn to the importance of CME-driven shocks for SEP acceleration: the shock accelerates and injects particles continuously as it propagates outward. It was then suggested that SEP events be classified into one of two categories: impulsive or gradual. In gradual events, particles are accelerated by the fast-mode CME shocks in the outer corona and in interplanetary space, while impulsive events are accelerated at the flare site/active region in the solar corona.

[7] Later observational evidence of the "mixed" events exhibiting distinct signatures of both impulsive and gradual events [e.g., Cohen et al., 1999, Cane et al., 2003, 2006] raised questions about the effectiveness of such a dichotomy in classifying the SEP events [Cliver, 2008, Kallenrode, 2003]. Kallenrode [2003] has suggested that pure impulsive and pure gradual events are only the extreme cases of SEP events and there is a continuous transition between different kinds of SEP events.

[8] Though the relative roles of the flare and CME in SEP acceleration remain an active research topic, mounting evidence indicates that the CME-driven shock plays a dominant role in generating large SEP events [e.g., Cliver and Ling, 2009; Gopalswamy et al., 2003; Tylka and Lee, 2006]. Being able to model a CME and its angular extent accurately will not only aid in assessing its impacts on a specific spacecraft or a region of interest, but also aid in estimating the spatial domain where an SEP event is expected to be observed, as illustrated by Figure 5 in Li and Zank [2005].

[9] This paper is organized as follows. Section 2 provides a brief description of the model used for modeling the evolution of CME(s) in interplanetary space - the coupled Wang-Sheeley-Arge (WSA)+ENLIL+Cone model. Section 3 describes what we have learned about accurately modeling CMEs and their impacts based on our space weather operations. Summary and discussion are presented in section 4 .

\section{The WSA+ENLIL+CONE Model}

[10] A number of models are available for simulating the propagation and evolution of CMEs in interplanetary space, each with its own strengths and weakness. For example, the coupled coronal (a resistive MHD model See Linker and Mikic [1995]) and heliospheric (a global heliospheric model called ENLIL) model suite described in Odstrcil et al. [2002] is capable of capturing many large-scale features of the solar corona and heliosphere. The model result was used to interpret the global context of CME observations by ACE at $1 \mathrm{AU}$ and Ulysses at $5 \mathrm{AU}$ [Riley et al., 2003]. Other CME models include the HAF (Hakamada-AkasofuFry) v2 [e.g., Wu et al., 1983; Fry et al., 2001] + 3-D MHD Model Ensemble code as described by $W u$ et al. [2011, and references therein] and the MHD model in Shen et al. [2011] and Zhou et al., [2012], the coupled WSA+ENLIL+Cone model [e.g., Odstrcil et al., 2004a, 2004b, 2005], the drag-based analytical CME propagation model [e.g., Vršnak et al., 2010], the Solar Corona, Eruptive Event Generator, and Inner Heliosphere modules of the Space Weather Modeling Framework (SWMF) [Tóth et al., 2005] and so on. It is not the intent of this paper to provide an exhaustive list of all the available CME models. The rationale for our model selection is discussed below.

[11] March 2010 marked the establishment of the Space Weather Research Center (SWRC) at NASA Goddard Space Flight Center (GSFC), a part of the Community Coordinated Modeling Center (CCMC, http://ccmc.gsfc.nasa.gov), with a primary objective of addressing the space weather needs of NASA's robotic missions through experimental research forecasts, notification, analysis, and education (http://swrc. gsfc.nasa.gov). The center represents maximal leverage of the latest scientific research results and more than a decade of modeling capabilities enabled by CCMC. SWRC aims to be a locus of innovation in research-based space weather analysis and forecasting prototypes. It not only addresses the unique space weather needs of NASA users, but also pioneers next generation space weather prediction systems. CCMCI SWRC differs from NOAA Space Weather Prediction Center (SWPC) in many aspects; one important difference is that CCMC/SWRC broadens the conventional space weather regime to include interplanetary space weather [Guhathakurta, 2013]; i.e., it monitors/tracks CMEs (together with other space weather effects) in all directions, while NOAA/SWPC is most concerned with Earth-directed eruptions and space weather effects in geospace. The disclaimer notice posted on the CCMC/SWRC website defines these different roles and points out that NOAA/SWPC provides the official U.S. Government space weather forecast.

[12] Taking into account previous research, model validation results, model performance [e.g., Odstrcil et al., 2004a, 2004b, 2005; Taktakishvili et al., 2009, 2011], and running time, our space weather operations use the coupled 
WSA+ENLIL+Cone model, which is also running at NOAA/SWPC [Pizzo et al., 2011]. As the most sophisticated model currently available to space weather forecasters, it is capable of providing a 1-2 day lead-time forecasting for major CMEs. Of course, as with all current models, it also has limitations as evidenced by discrepancies when compared with observations. The discrepancies can be attributed to multiple factors, including the idealistic "cone" representation of CMEs in the model and the absence of an internal magnetic structure in the modeled CMEs. The latter is a major limitation of almost all models; adding the CMEs' internal structure remains both physically challenging and computationally expensive. It should be stressed that the focus of this paper is not on fine tuning the model inputs to produce the best agreement with observations, but rather on what has been learned by performing real-time CME runs.

[13] The Wang-Sheeley-Arge (WSA) model [Schatten, 1971; Wang and Sheeley, 1995] is a combined empirical and physics-based representation of the quasi-steady global solar wind flow that can be used to predict ambient solar wind speed and Interplanetary Magnetic Field (IMF) polarity at Earth. The coronal field is determined by a potential field source-surface calculation, for which the inner boundary is specified with the use of synoptic magnetograms [Arge and Pizzo, 2000]. WSA computes the solar wind speed at the source surface using an empirical relationship. Global Oscillation Network Group (GONG) solar magnetograms are used as input for the WSA model in our operation.

[14] ENLIL is a time-dependent, 3-D ideal MHD model of the solar wind in the heliosphere [Odstrcil et al., 2002, 2004a, 2004b], designed to treat supersonic outflows in the limit where resistivity and viscosity are minimal. It is based on the polytropic equation of state. The ENLIL model domain extends from the solar equator to within $\pm 60^{\circ}$. This grid has sufficient latitudinal range to minimize the effects of neglecting high-latitude behavior on low latitudes [Odstrcil and Pizzo, 1999].

[15] The cone model concept (similar to Zhao et al. [2002] and Xie et al. [2004]) is used to model a CME and its propagation through interplanetary space, assuming that it propagates with nearly constant angular width in a radial direction and that the expansion is isotropic. Although rather simplistic, it has been reasonably successful in representing CMEs and their impacts at points of interest in interplanetary space [Taktakishvili et al., 2011; Falkenberg et al., 2010; 2011] in combination with the WSA+ENLIL model.

[16] In the coupled WSA+ENLIL+Cone approach [Odstrcil et al., 2004a, 2004b], output from the WSA is used to set up the ENLIL inner boundary condition at $21.5 \mathrm{Rs}$ (solar radii) so that relatively realistic solar coronal magnetic field and structures are taken into consideration. ENLIL, together with the Cone model, accounts for the ambient solar wind, transient CME structures, and their interaction and propagation in the simulation domain of the heliosphere. The modeling results from the combined CME model are not only useful for forecasters, but also valuable for interpreting scientific results [e.g., Riley et al., 2003; Odstrcil et al., 2004a, 2004b; Baker et al., 2013; Mewaldt et al., 2013].

[17] The main input parameters for starting the WSA +ENLIL+Cone model are the kinematic parameters of CMEs, which are obtained primarily from the white-light coronagraph images provided by SOHO, STEREO A, and STEREO B spacecraft. A robust triangulation method, evolved from the one described in Pulkkinen et al. [2010] and based on the projection matrix formulation, is used to determine the kinetic properties of a CME. The method and the corresponding triangulation tool were developed for quick and easy use in an operational space weather forecasting setting. Coronagraph images from at least two spacecraft are needed for the triangulation method. During a specified interval of interest, at least two images from each of the two spacecraft are needed to derive the CME parameters. The leading edge of a CME image is used to derive CME speed. The details of the method will be reported elsewhere.

[18] The input parameters for the Cone model include the propagation direction of a CME in terms of longitude and latitude in Heliocentric Earth Equatorial (HEEQ) coordinates, its speed, its angular width, and the time of its arrival at 21.5 Rs (ENLIL's inner boundary).

\section{CME Forecasting in an Operational Setting}

[19] There are a number of challenges that need to be dealt with in a real-time setting as opposed to postevent $\mathrm{CME}$ analysis. Due to the fact that the coronagraph images are all from science missions (SOHO, STEREO A, and STEREO B), and there are data downlink limitations, data gaps in real-time coronagraph images are to be expected. In addition, the (near) real-time coronagraph beacon images on STEREO A and B are compressed to minimize the data downlink size so an inferior quality of images is often encountered in an operational setting, although many of these problems can be alleviated when dealing with science quality data for research purposes. The limitations and constraints of real-time observations and the desire to achieve accurate forecasting of CMEs necessitate exploiting and synthesizing all information sources to better assess CME characteristics. The example below illustrates the importance of utilizing a CME's 3-D properties in forecasting CME impacts.

\subsection{The Importance of Synthesizing All Available Information}

[20] To improve the forecast of CME propagation/evolution, it is necessary to synthesize all data sources available to extract as much information as possible. For example, the (near) real-time SDO (Solar Dynamic Observatory) (http://sdo.gsfc.nasa.gov) images at high spatial and temporal resolution, particularly the AIA data in different EUV wavelengths, have been invaluable in our operation, allowing us to look at the CMEs in different "lights" (different EUV wavelengths in addition to the white-light 
Table 1. Parameters of the CME That Occurred on 4 October 2011

\begin{tabular}{lcccr}
\hline CME Starting Time & Speed km/s & Direction LON/LAT in HEEQ & Half-Cone Angle (Degrees) & Time at 21.5 Rs \\
\hline 2011-10-04 13:10 UT & 1370 & $-155 / 35$ & 50 & $2011-10-04$ 15:23 UT \\
\hline
\end{tabular}

coronagraph images) and providing valuable additional information for improving the $\mathrm{CME}$ input parameters needed for the global Heliospheric model. Similarly, the EUV images from STEREO A and B are often used to aid our operation (particularly for farside CMEs), along with many other types of observations, such as radio emissions. Recent research results have shown that CMEs can change direction during their propagation [Byrne et al., 2010]. During our operations, several CMEs originating from a high-latitude active region were found to deflect toward the ecliptic plane. A systematic effort is under way to synthesize all information sources in order to better determine CME input parameters (especially when coronagraph images are only available from one spacecraft), and thus further improve our forecasts.

[21] As described by Cane and Lario [2006], energetic particle observations in interplanetary space can be used to probe the origin, development, and structure of coronal mass ejections. The example below shows how we used the concurrent SEP observations (in combination with others) to assist our CME impact forecast.

[22] Table 1 lists the input parameters for the CME that occurred on 4 October 2011. This is a backsided, fairly high-latitude $\left(35^{\circ}\right) \mathrm{CME}$ with a fast speed $(\sim 1370 \mathrm{~km} / \mathrm{s})$ and a broad angular width starting around 4 October 2011 13:10 UT. Enhancement in energetic particle fluxes was detected over a broad longitudinal extent as evidenced in Figure 1: both STEREO A and STEREO B saw flux increases in the $13-100 \mathrm{MeV}$ protons following the onset of the CME. The MESSENGER spacecraft in the vicinity of Mercury also detected enhancement in SEP fluxes. Because particles tend to follow magnetic field lines, they can be used to trace field line topologies. Also, shock-accelerated populations provide information about the size of a CME shock as it propagates from the Sun to the observer.

[23] Panels (a) and (b) of Figure 2 provide the simulated three-dimensional density map of the solar wind at 7 October 2011 06:00 UT (interchange with 7 October 2011 T06:00Z) and 12:00 UT respectively. The solar wind density distribution from three different viewing perspectives is displayed, with the left panel providing a view of the ecliptic plane, the middle panel showing the meridian plane cutting through the Sun-Earth line, and the right panel showing the Mercator projection of the $1 \mathrm{AU}$ (Astronomical Unit) spherical surface (the horizontal axis showing the latitude and the vertical axis showing the longitude). Our simulation results indicate that the CME may barely touch STEREO B (the automatic routine indicated a non-encounter with STEREO B). In addition, its relatively high-latitude $\left(35^{\circ}\right)$ propagation also acts against its potential impact on STEREO B, which lies close to the ecliptic plane. However, the observed flux enhancement in the $13-100 \mathrm{MeV}$ energy range by STEREO $B$ shortly after the onset of the CME suggests strongly that there is a good magnetic connectivity of the CME shock and STEREO $B$ and the size of the CME shock is broad enough to affect STEREO B. By synthesizing all this information (both from model simulations and observations), we issued our CME alert (http://1.usa.gov/1f1fEjp) and provided an estimated arrival time for both MESSENGER and STEREO B. In the alert, we stated that "Simulations indicate that the leading edge of the CME will reach STEREO $B$ around 2011-10-07T06:00Z (plus minus 7 hours) - the flank of the CME" (Figure 2 indicates that a more accurate time would be somewhere in between 06:00 UT and 12:00 UT). Our model estimate was close to the actual shock arrival around 7 October 2011 11:30 UT, as indicated by the in situ plasma and IMF measurements onboard STEREO B (See Figure 3, quantities from STEREO B are in blue).

\subsection{Added Benefit of the CME Simulation Results for SEPs}

[24] An active day in terms of solar activities was 7 March 2011 with occurrences of $5 \mathrm{M}$-class flares and two significant CMEs. The two significant CMEs are associated with the M1.9 class flare, which peaked at 14:30 UT (starting at 13:45 UT, peaking at 14:30 UT and ending at 14:56 UT) from the Active Region 11166 (N11E21) and the M3.7 class flare, which peaked at 20:12 UT (starting at 19:43 UT, peaking at 20:12 UT and ending at 20:58 UT) from the Active Region 11164 (N23W50), respectively. Type II and Type IV radio emissions were also observed with the M1.9 flare at 14:30 UT, while the Type II radio emission and a Tenflare (strong emissions in the $10.7 \mathrm{~cm}$ wavelength band) were detected in association with the M3.7 flare.

[25] Figure 4 shows the real-time SDO EVE (Extreme Ultraviolet Variability Experiment) [Woods et al., 2010] images of the two flares at times close to their peak. Although the quality of these images is not superlative, they provide quick visual information on where a flare/ CME originates, and are thus useful for space weather operations. The location of a flare/CME can help with initial assessment of their potential space weather impact as past statistical results [e.g., Gopalswamy et al., 2005c] indicate that the geoeffective CMEs tend to occur within $30^{\circ}$ of the center meridian, while Earth-impacted SEP events tend to favor the vicinity of $60^{\circ}$ west (the right side on the images) in longitude. The space weather impacts of the two flares/CMEs conform to the statistical results (more details are provided below), with the first CME being more geoeffective and the second CME (and its associated flare) being more SEP effective at Earth and is unlikely to have strong geomagnetic storm effects. 

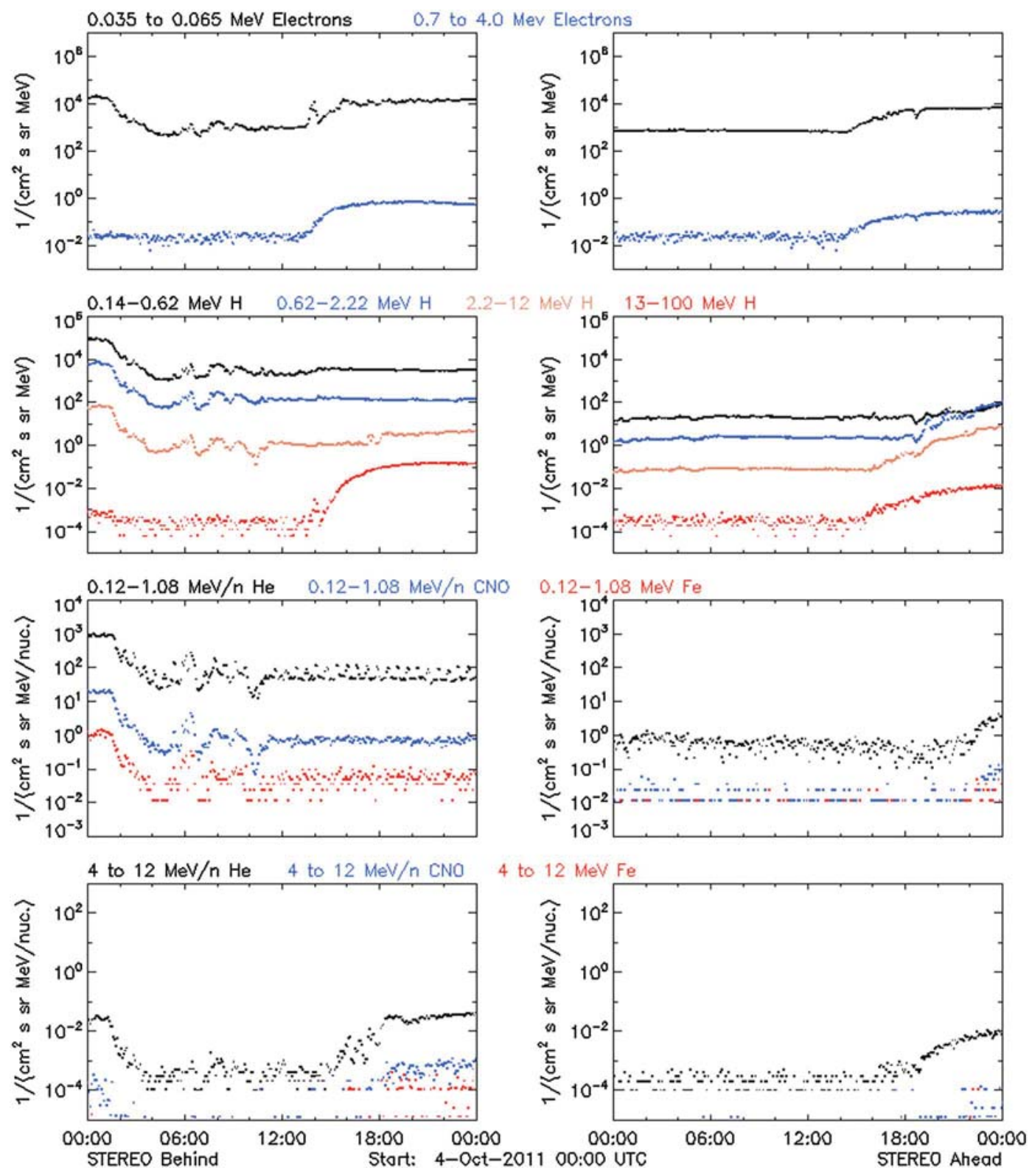

Figure 1. STEREO beacon data of energetic particle flux at different energy channels, with the left side ones from STEREO B and the right side from STEREO A.

[26] Figure 5 shows the locations of the STEREO spacecraft (B for STEREO B and A for STEREO A). SOHO is located at the L1 Lagrangian upstream location along the Sun-Earth line, about $1 / 100^{\text {th }}$ of the distance from Earth to the Sun. Figure 6 contains the coronagraph images for two CMEs. The top images are the snapshots of the first CME around 7 March 2011 17:00 UT viewed from three different spacecraft: STEREO B, SOHO, and STEREO A. The bottom row shows the snapshot of the second CME around 7 March 2011 21:00 UT from three different viewpoints. The time sequence of these coronagraph images from three spacecraft was used (together with the ephemeris information of the spacecraft) to extract kinetic CME parameters that are needed for the WSA+ENLIL+Cone model.

\subsubsection{Nominal Use: Time of Arrival Prediction and} Impact Assessment

[27] The Cone model input parameters for the two CMEs are listed in Table 2. In the WSA+ENLIL+CONE model suite, CME density can be modified according to each individual CME. But operationally, we use the default setting where the CME density is four times of the ambient solar wind. The reason is that determining CME density from real-time data streams remains difficult in comparison with the other parameters mentioned above. This set of 

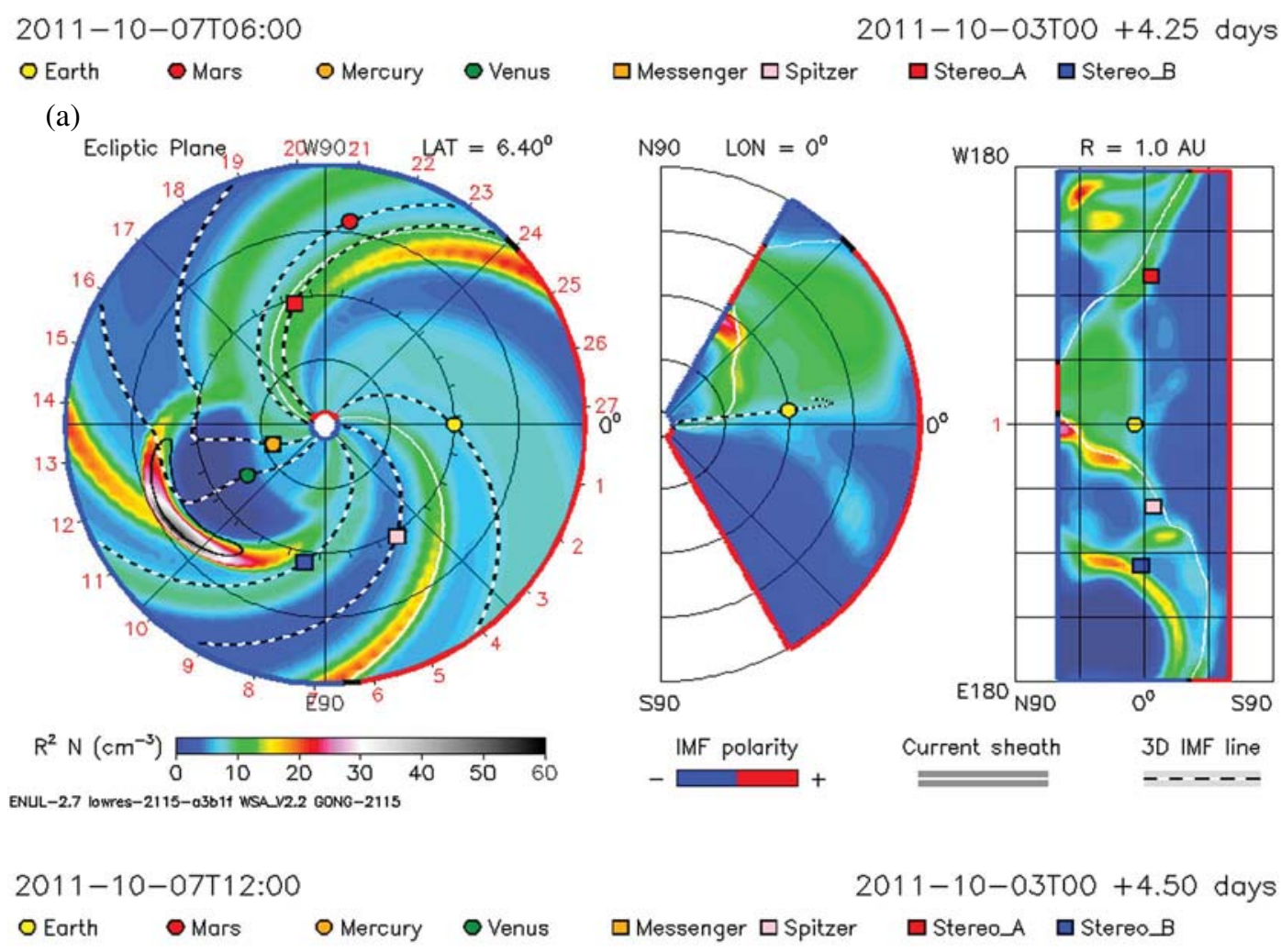

(b)
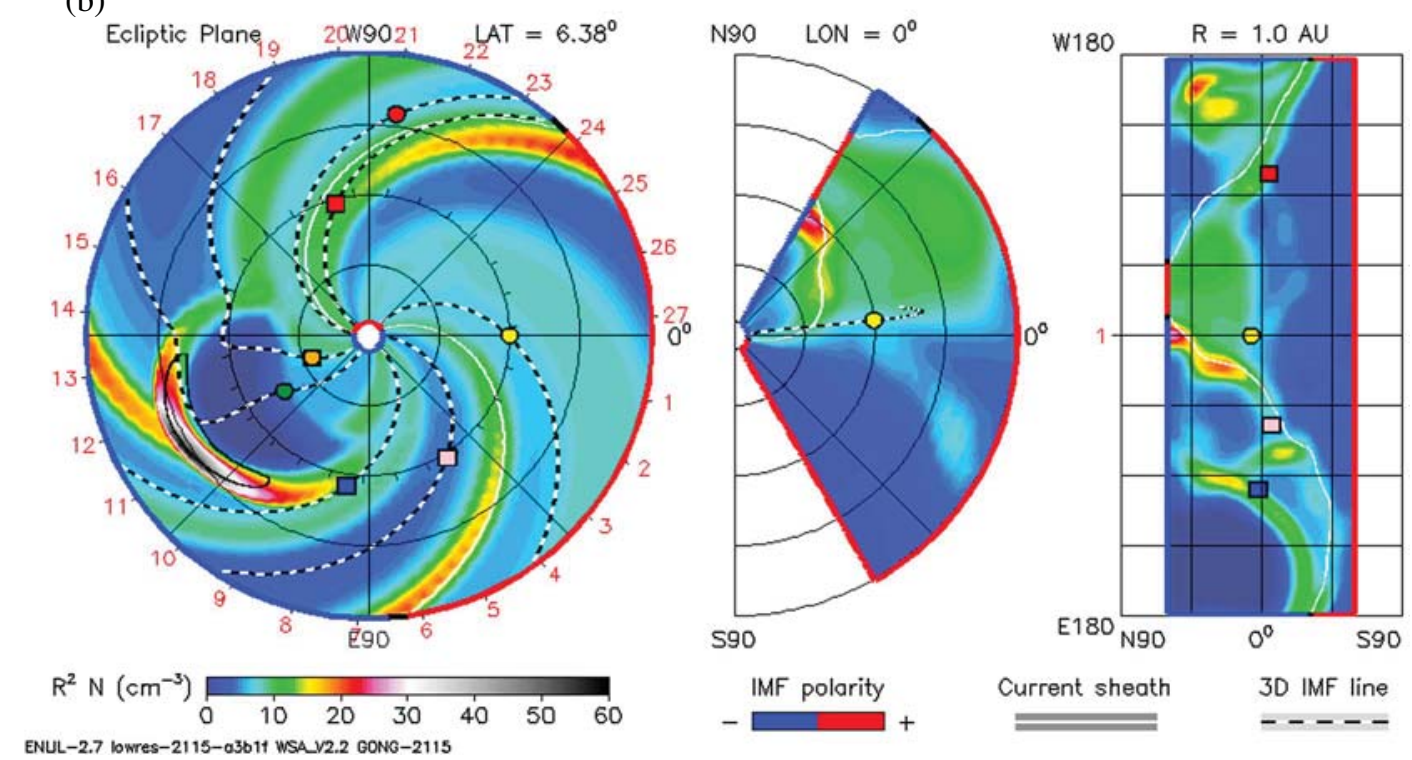

Figure 2. (a) The simulated 3-D solar wind density map at 7 October 2011 06:00 UT. (b) The simulated 3-D solar wind density map at 7 October 2011 12:00 UT.

parameters is the same one we used for our space weather operations. The simulation results were communicated to our users at near real time (normally with a couple of hours delay due to the waiting time for enough coronagraph images available for analysis and another $\sim 20 \mathrm{~min}$ for the model execution. The 1-4 days lead-time forecasting capability from the WSA+ENLIL+Cone model still makes the effort worthwhile). They are not adjusted/fine-tuned to match the observational results.

[28] The temporal evolution of the solar density, solar wind speed from the ENLIL+WSA+Cone modeling results (dashed lines) at L1 (around Earth) and STEREO A is shown in Figure 7, along with the observations (solid lines), with Figure $7 \mathrm{a}$ showing those at Earth and Figure $7 \mathrm{~b}$ 


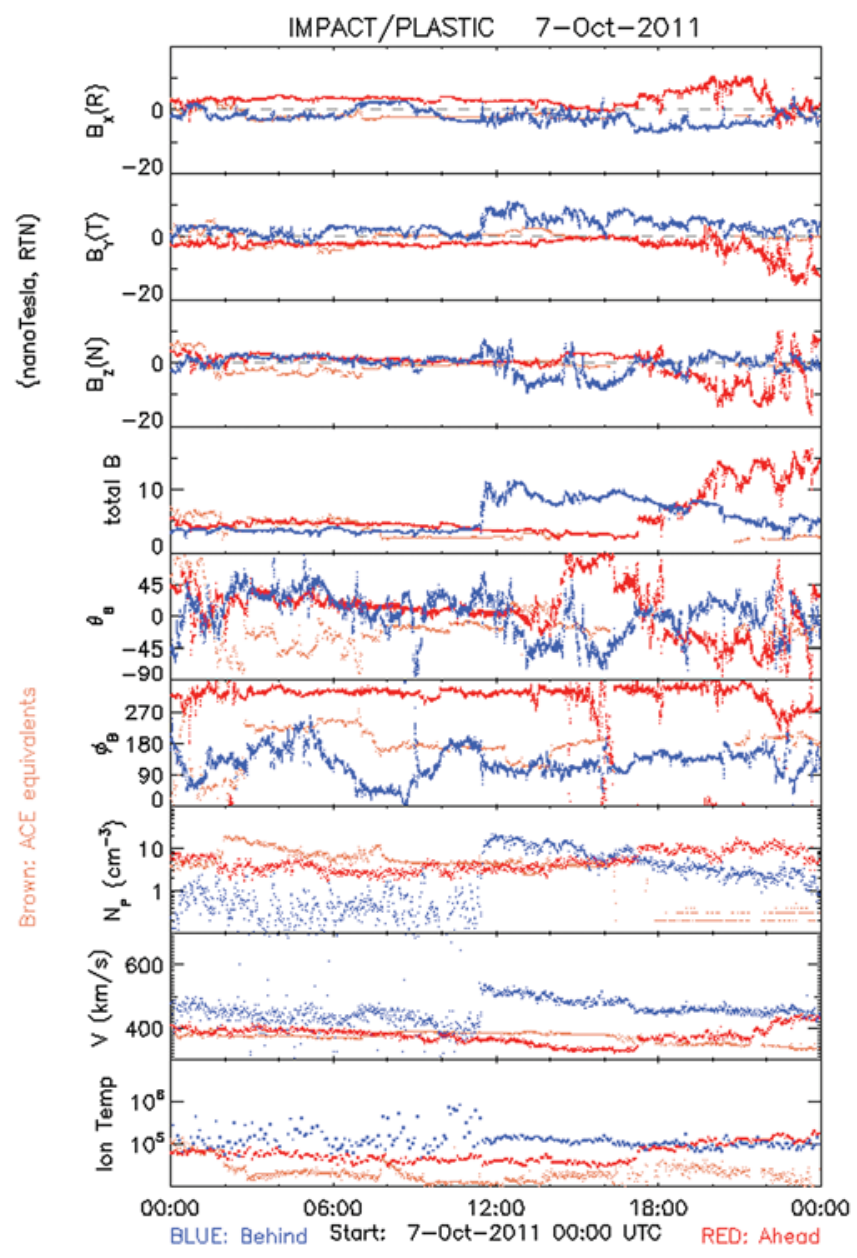

Figure 3. In situ beacon data of solar wind plasma and IMF from STEREO B (blue) and STEREO A (red). Note the shock arrival at 11:30 UT detected by STEREO B.

showing those at the STEREO A location. The vertical dashed lines indicate the observed shock arrival time at L1 or STEREO A, while the dotted vertical lines are the corresponding simulated arrival time.

[29] It should be reiterated that unlike research studies, the simulation results shown here (and throughout this paper) are from real-time operations (using the default model setting), which do not favor the fine tuning of various modeling parameters/settings to match the observations. For example, the model does not reproduce the first density peak at STEREO A on 8 March, which may have potentially slowed down the arrival of the CME at STEREO A and explain the earlier simulated arrival time in comparison with the observed one. In addition, at L1, the shock structure from the simulation is more gradual than ACE's observations.

[30] From an operational perspective, however, the forecast arrival time at Earth (L1) and STEREO A was reasonably accurate. ACE observed an interplanetary shock at 10 March 2011 06:00 UT (the dashed vertical line in Figure 7a), while our predicted arrival is around 10 March 2011 06:52 UT (the dotted vertical line in Figure 7a). Similarly, STEREO A observed an interplanetary shock at 9 March 2011 06:50 UT as indicated by the IMPACT (In situ Measurements of Particles and CME Transients)/PLASTIC (PLAsma SuperThermal Ion Composition) beacon data (the dashed vertical line in Figure $7 \mathrm{~b}$ ), while our predicted arrival is around 9 March 2011 00:45 UT (the dotted vertical line in Figure $7 \mathrm{~b})$. Both are within the statistical error $( \pm 7 \mathrm{~h})$ derived from the geoeffective historical events [Taktakishvili et al., 2009]. Model results, it should be noted, usually lack a clear distinction between the shock and the CME material itself. The arrival time in our operation is defined when the time derivative of the modeled dynamic pressure exceeds a certain threshold value.

[31] As a part of our CME forecasting, we also estimate the maximum value of $\mathrm{Kp}$ (a geomagnetic activity index, ranging 0-9) if a CME(s) heads toward Earth. Although $\mathrm{Kp}$ is a rather simple and low temporal cadence index, it is used widely in operational space weather. The Kpmax is derived based on Newell et al. [2007] formula using the
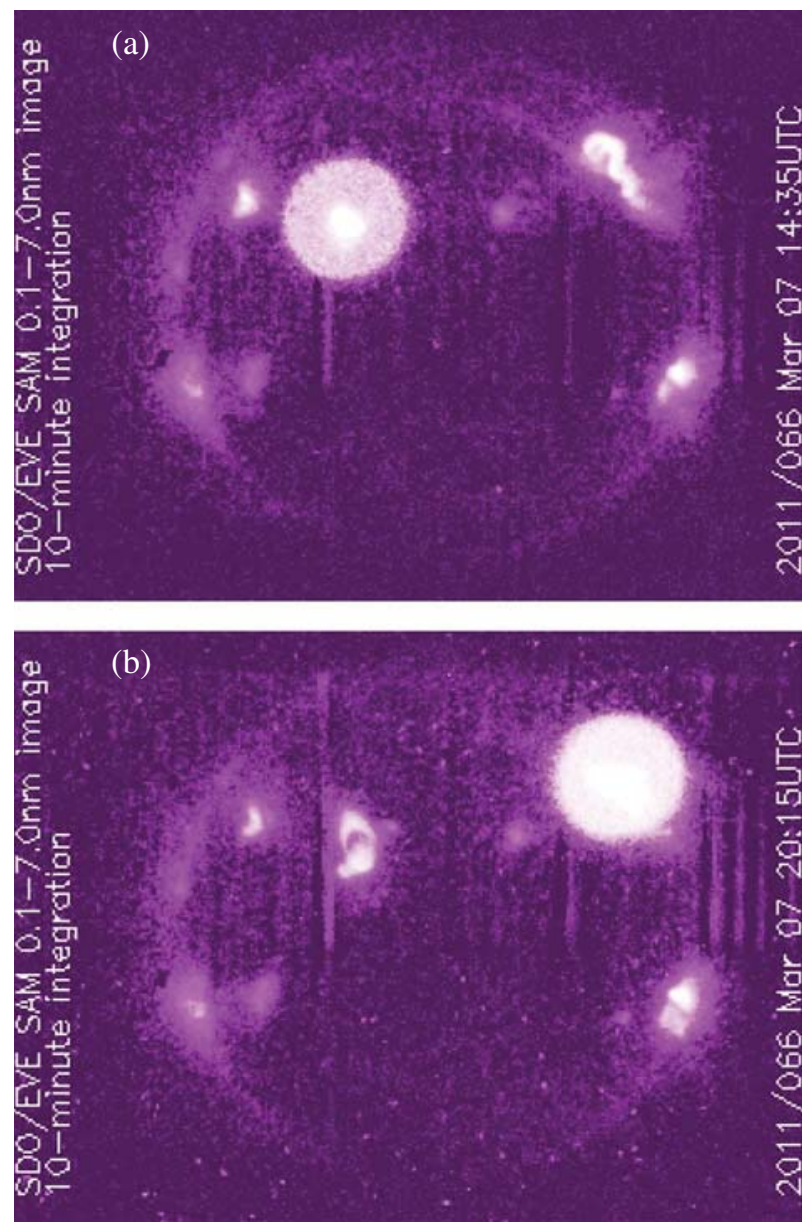

Figure 4. SDO EVE images of the (a) M1.9 and (b) M3.7 flare at times close to their peak intensity. 


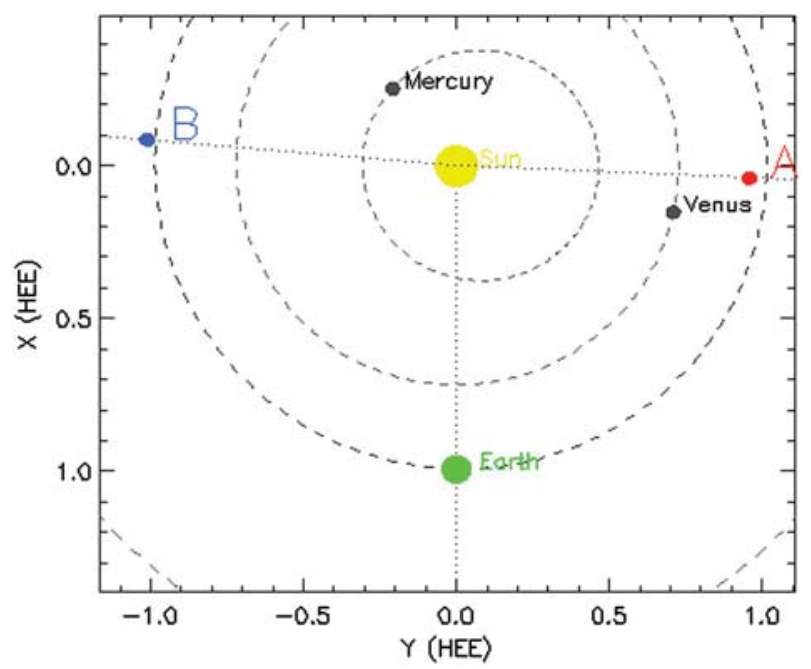

Figure 5. Locations of STEREO A and STEREO B in HEE (Heliocentric Earth Ecliptic) coordinates in reference to the Sun. The SOHO spacecraft is very close to Earth in this picture, 1/100 th distance of the total SunEarth distance. Courtesy of the STEREO orbit tool at http://stereo-ssc.nascom.nasa.gov/where/. modeled solar and IMF conditions. Since WSA+ENLIL +Cone does not give the polarity of the field in a realistic manner for a $\mathrm{CME}(\mathrm{s})$, we assume variety of IMF clock angles. Kpmax results by assuming the maximum of the modeled field to be southward. For the run above, the estimated $\mathrm{Kp}$ is 4 (same as what was observed).

[32] While the above example demonstrates the default usage of the WSA+ENLIL+Cone simulation results, next we will show a rather novel usage of the CME simulation results, which provide a qualitative and graphical estimate of SEP distributions that have proven to be useful/informative for our NASA mission operators.

3.2.2. CME Simulation Results: Visual Representation of SEP Distribution

[33] From a space weather perspective, another interesting aspect of the 7 March event is that an enhancement of solar energetic particle (including electrons and ions) fluxes was observed across a broad longitude in space, at spacecraft along the Sun-Earth line by SOHO and ACE (L1), and GOES at geosynchronous orbit, and at STEREO A. This SEP event is associated with the M3.7 flare (the flare peak time is marked by the left dashed line in Figure 8a) and the $1980 \mathrm{~km} / \mathrm{s}$ CME. Figure 8 shows the temporal profile of fluxes at different energy levels from different spacecraft.
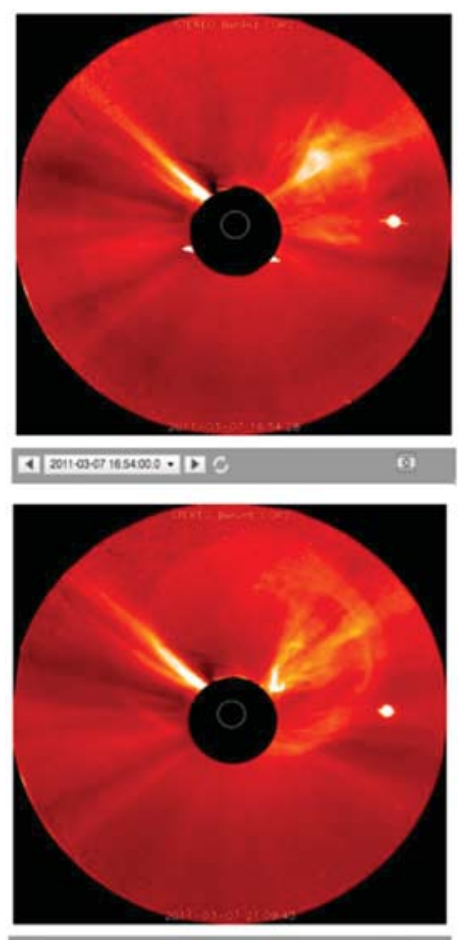

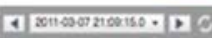
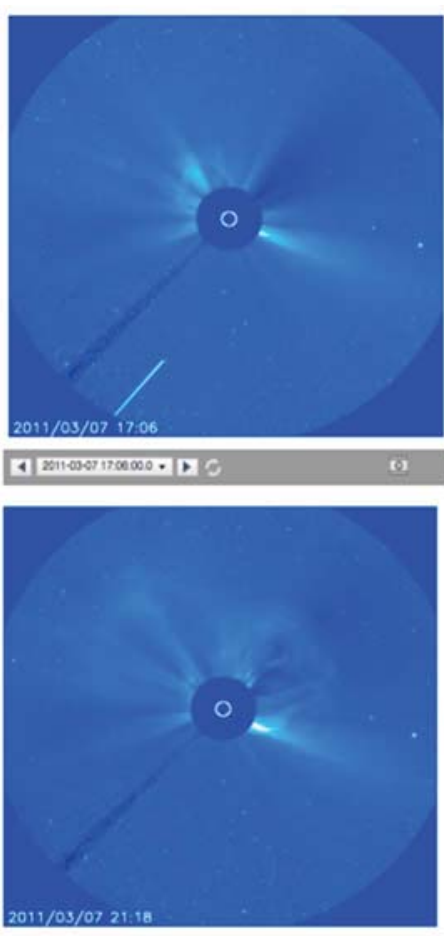

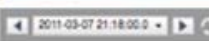
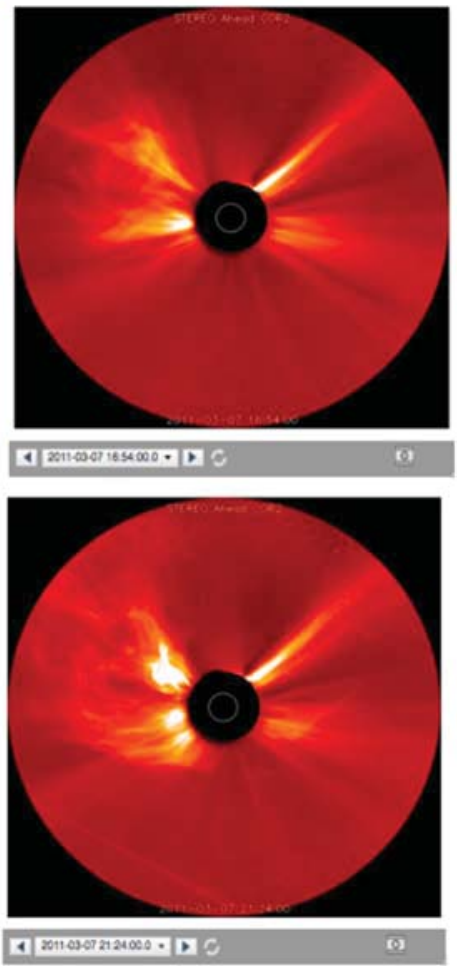

Figure 6. Coronagraph images for two CMEs. The top images are the snapshot of the first CME around 7 March 2011 17:00 UT viewed from three different spacecraft: STEREO B, SOHO, and STEREO A. Similarly, the bottom row shows the snapshot of the second CME around 7 March 2011 21:00 UT from three different vantage points. 
ZHENG ET AL.: FORECASTING CMES

Table 2. The Cone Model Input Parameters for the Two CMEs on 7 March 2011

\begin{tabular}{lcccr}
\hline CME \# Associated Flare & Speed km/s & Direction LON/LAT in HEEQ & Half-Cone Angle (Degrees) & Time at 21.5 Rs \\
\hline M1.9 flare 14:30 UT & 710 & $-13 / 15$ & 35 & $2011-03-07$ 19:51 UT \\
M3.7 flare 20:12 UT & 1980 & $50 / 17$ & 45 & $2011-03-0721: 40$ UT \\
\hline
\end{tabular}

Figure 8a displays those from STEREO A, ACE, and GOES in reference to the solar flare intensity in two different $X$ ray bands in units of watts $/ \mathrm{m}^{2}$ (the 1-8 A wavelengths in black and the $0.5-4 \mathrm{~A}$ in blue at the bottom of the plot). Figure $8 \mathrm{~b}$ shows energetic proton fluxes measured by SOHO/COSTEP (COmprehensive SupraThermal and Energetic Particle Analyzer).

[34] For Figure $8 \mathrm{a}$, the dark red line is the $38-53 \mathrm{keV}$ electron differential flux (unit: $1 / \mathrm{cm}^{2} / \mathrm{s} / \mathrm{sr} / \mathrm{MeV}$ ) from ACE, the green line is the $35-65 \mathrm{keV}$ electron differential flux (unit: $1 / \mathrm{cm}^{2} / \mathrm{s} / \mathrm{sr} / \mathrm{MeV}$ ) from STEREO A, the purple line is the $>10 \mathrm{MeV}$ proton integral flux from ACE (unit: $1 / \mathrm{cm}^{2} / \mathrm{s} / \mathrm{sr}$ ), the red line is the $>10 \mathrm{MeV}$ proton integral flux from GOES 13 (unit: $1 / \mathrm{cm}^{2} / \mathrm{s} / \mathrm{sr}$ ), the black line is the 2.2-12 MeV proton differential flux from STEREO A (unit: $1 / \mathrm{cm}^{2} / \mathrm{s} / \mathrm{sr} / \mathrm{MeV}$ ), and the yellow line is the $13-100 \mathrm{MeV}$ proton differential flux (unit: $1 / \mathrm{cm}^{2} / \mathrm{s} / \mathrm{sr} / \mathrm{MeV}$ ) from STEREO A. For Figure $8 \mathrm{~b}$, the black line is the $4-9 \mathrm{MeV}$ proton differential flux, blue line for the $9-15.8 \mathrm{MeV}$ proton flux, light orange for the $15.8-39.8 \mathrm{MeV}$ proton flux, and dark orange for the $28.2-50.1 \mathrm{MeV}$ proton flux, all of which are in the unit of $1 / \mathrm{cm}^{2} / \mathrm{s} / \mathrm{sr} / \mathrm{MeV}$.

[35] We can see that the SEP event has the characteristics of a gradual event in which the CME plays a significant role [e.g., Luhmann et al., 2007; Liou et al., 2013]. The energetic proton fluxes on STEREO A, in particular, show continuous increases as the CME-induced shock and ejecta move out toward STEREO A. When the shock/CME arrived at STEREO A, there was a reduction in SEP event intensity (indicated by the right dashed vertical line on Figure 8a) as if the strong magnetic fields in the CME ejecta form a barrier to particles' entry into that structure, often referred as the Forbush decrease [e.g., Cane, 2000].

[36] Due to complex physical processes and the different makeup (both in composition and energy spectrum) of SEP sources (including both flare and CME/ICME shock contributions) involved in different SEP events, a realistic SEP model capable of forecasting/modeling SEP event occurrence in three-dimensional interplanetary space (applicable for different spatial locations) and at the same time of operational use (computationally feasible) remains elusive [Luhmann et al., 2010; Zank et al., 2007]. The simulation results from the combined WSA+ENLIL+Cone model can be very useful in terms of qualitatively/visually providing an estimate of where an SEP event would be expected. The activity location plus the simulated CME/shock profile (both spatial and temporal) provide information on the magnetic connectivity to an observer and therefore facilitate a rough estimate of the expected SEP spatial extent. This approach assumes that the influence of the shock evolution dominates over the diffusive transport in determining the SEP profiles as shown by Luhmann et al. [2010]. Figure 9 serves as such an example, showing the solar wind density distribution from three different viewing perspectives. The "**"s and " $x$ "s indicate where the SEPs can be expected based on the simulated CME/shock properties and magnetic connectivity (the black and white dashed lines are magnetic field lines), which are consistent with the SEP observations at STEREO A and along the Sun-Earth line very well.
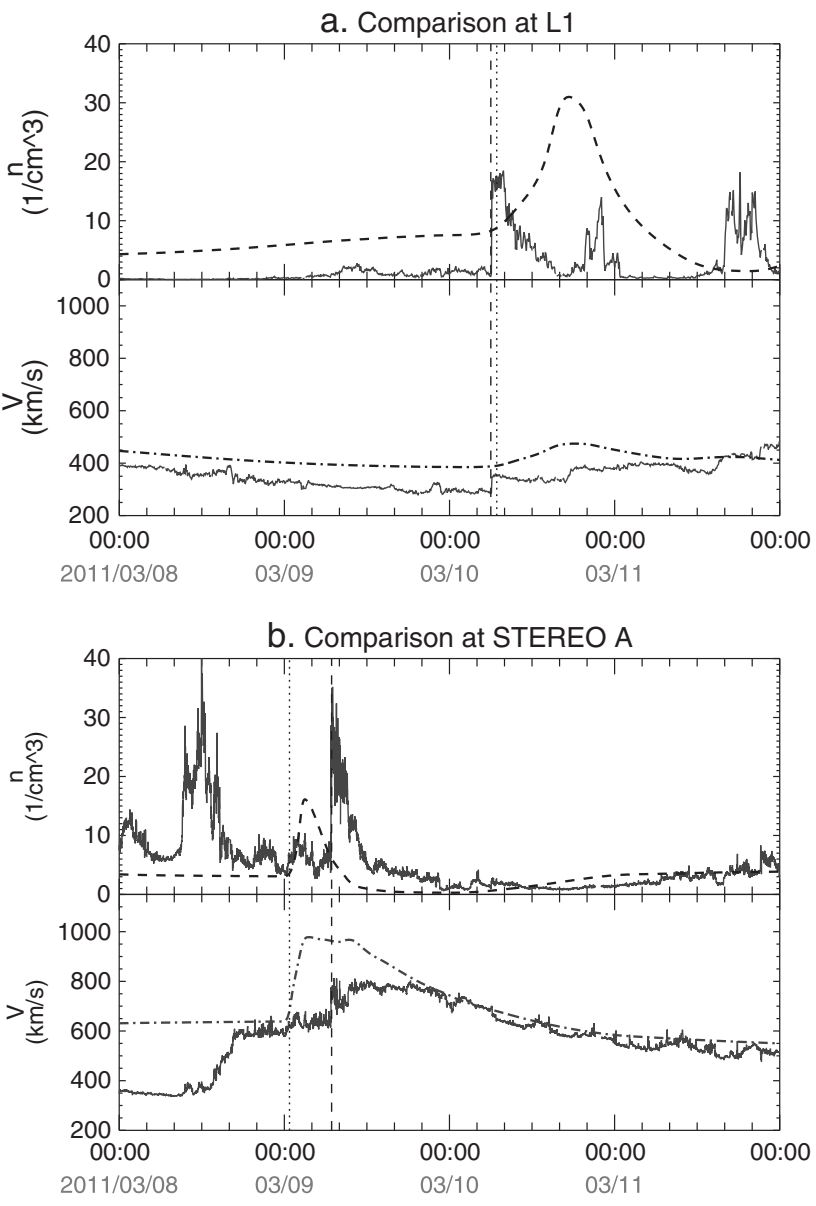

Figure 7. Simulated timeline of solar wind density and speed at L1 and STEREO A locations (dashed lines) and comparisons with observations (solid lines) (Run 1). The dashed line in the top panel of Figures $7 a$ and $7 b$ represents the density and the dot-dashed line represents the speed. The vertical dashed lines indicate the observed shock arrival time at L1 or STEREO A, while the dotted vertical lines are the corresponding simulated arrival time. 


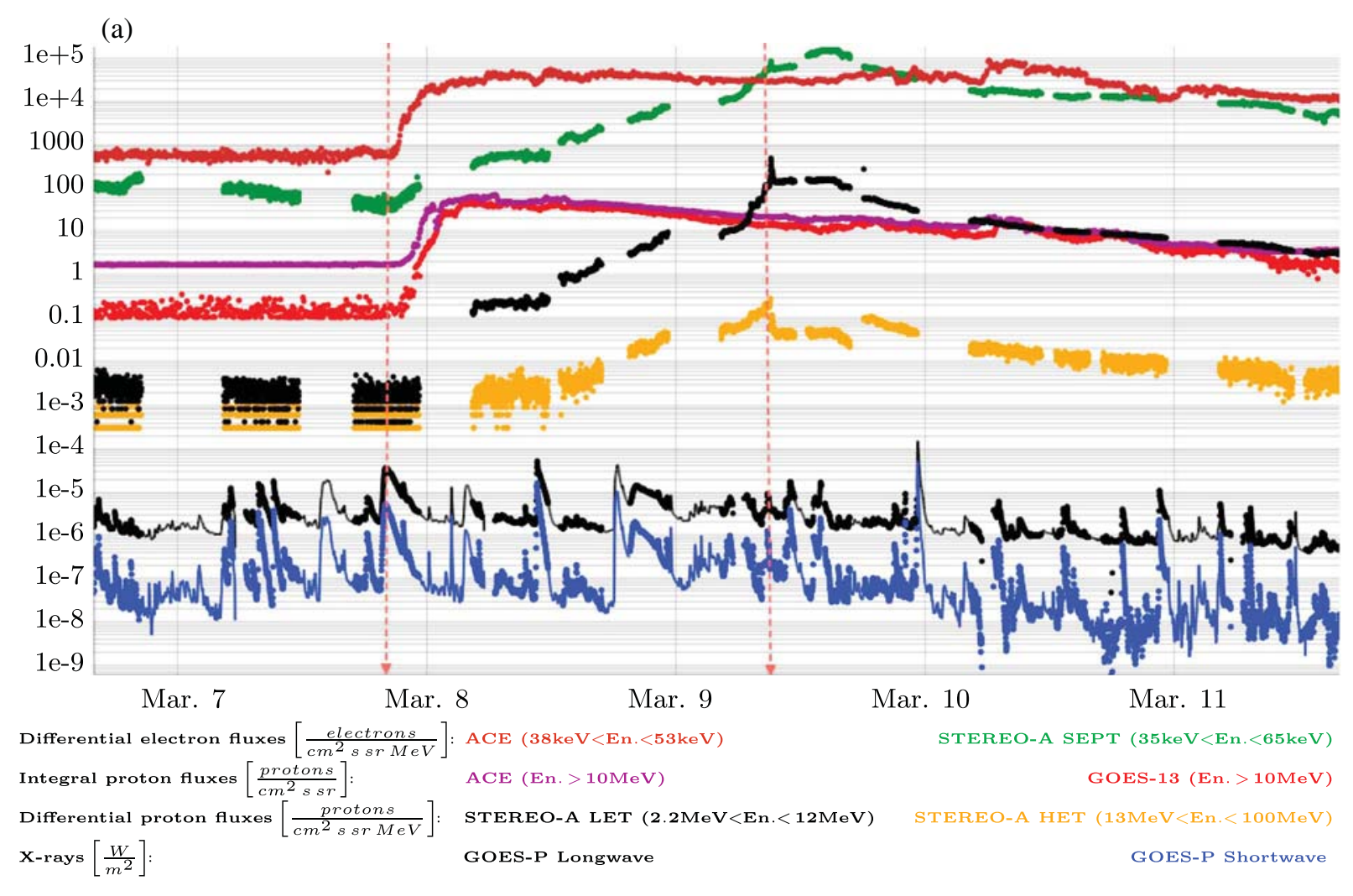

(b)

\section{SOHO/COSTEP real-time proton flux at CCMC data gaps due to limited DSN coverage}

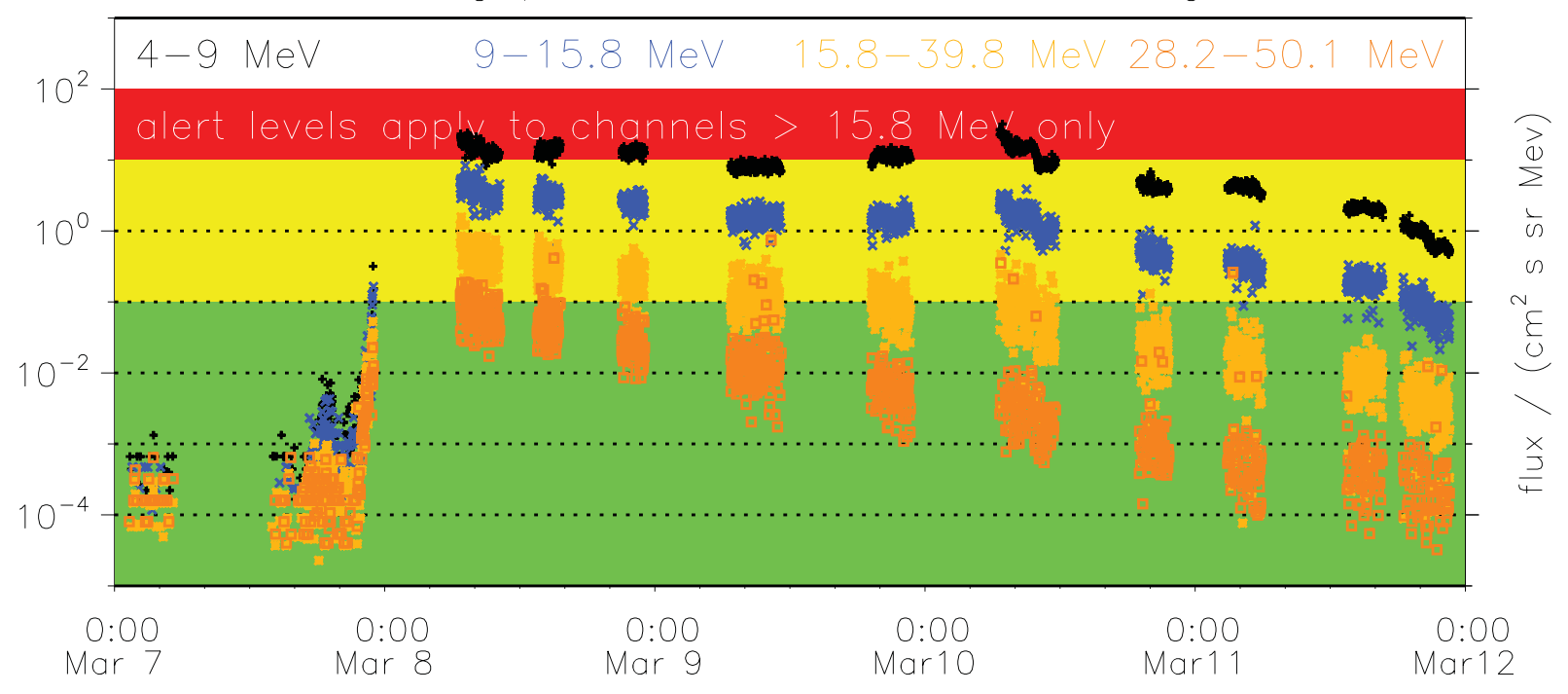

Figure 8. (a) SEP measurements from STEREO A, ACE, and GOES 13 in reference to the solar flare X-ray fluxes. (b) SEP measurements from SOHO at different energy channels.

\subsection{The Importance of Including All CMEs}

\section{in Quick Succession}

[37] Our operational experience shows that it is important to include all CMEs occurring in quick succession (e.g., within a $24 \mathrm{~h}$ period), especially for those CMEs which overlap in their propagation paths. The inclusion of all CMEs is to ensure realistic representation of interactions between CMEs and the ambient solar wind and interactions among 

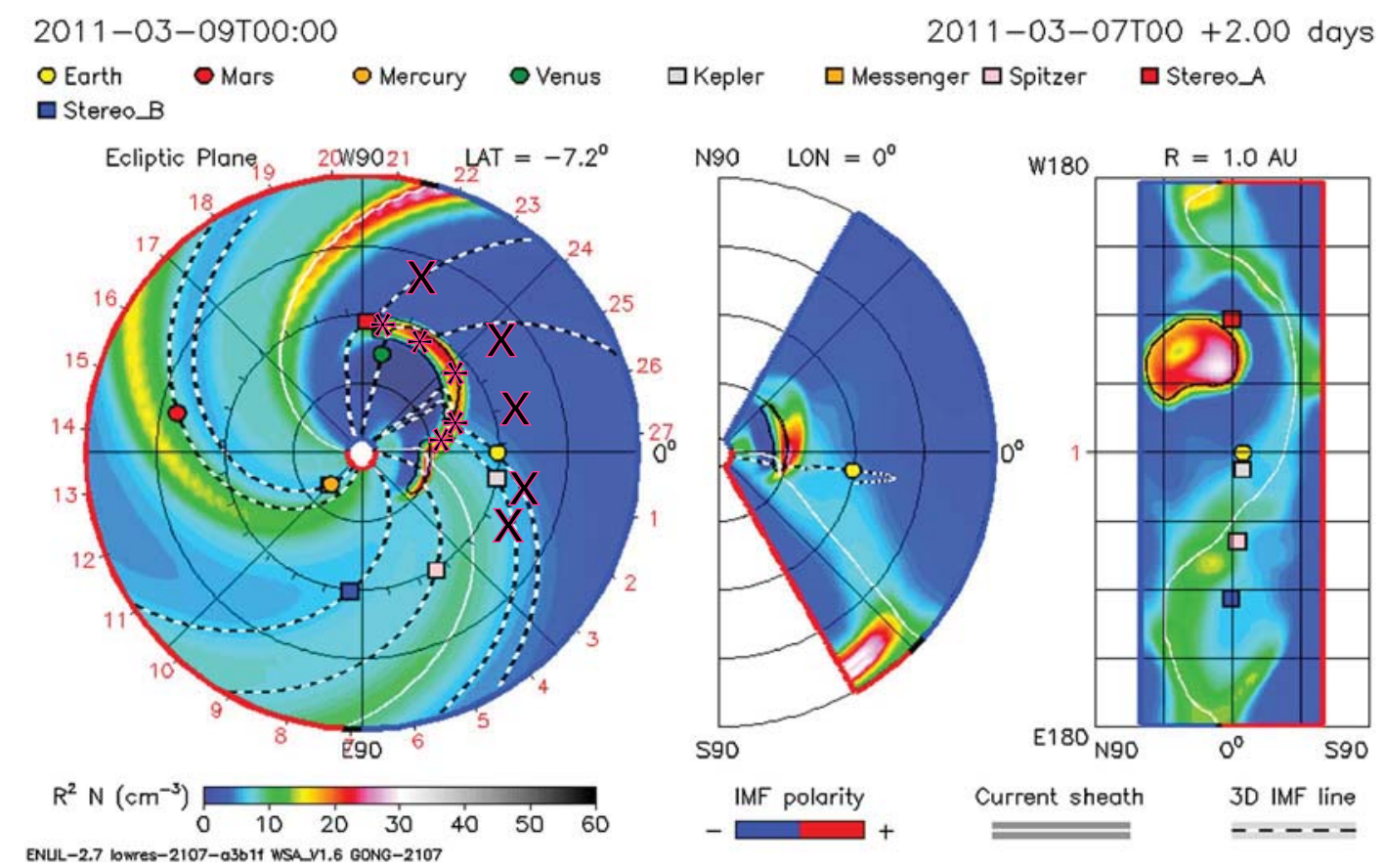

Figure 9. An example showing that the combined WSA + ENLIL + Cone simulation results can be used for SEP monitoring/forecasting.

CMEs themselves. An accurate capture of aforementioned interactions is important for forecasting CME arrivals and impacts.

[38] Within roughly a $24 \mathrm{~h}$ period during 18 January 2012 and 19 January 2012, three CMEs occurred. Details are listed in Table 3 in a sequential order. Since CME No. 2 is slow $(372 \mathrm{~km} / \mathrm{s})$ and narrow (half-cone angle of $27^{\circ}$ only), its impact is negligible in comparison with the other two (at a speed of $520 \mathrm{~km} / \mathrm{s}$ and $1020 \mathrm{~km} / \mathrm{s}$, respectively). However, it is still included in the simulation results shown below.

[39] Two runs were carried out during our space weather operation, with Run 1 including the two CMEs (No. 2 and No. 3) on 19 January 2012 and Run 2 including all three CMEs including the one on 18 January 2012 (No. 1, No. 2, and No. 3). Figure 10 shows the snapshots (at 20 January 2012T06:00Z) of the two WSA+ENLIL+Cone runs while still at the relatively early stage of the CMEs' evolution/ propagation, while Figure 11 shows the snapshots of the two runs when the CMEs were at the vicinity of Earth (at 22 January 2012T00:00Z). Figures 10 and 11 bear the same format as Figures 2 and 9, illustrating the rather standard density distribution in three different cut planes.
[40] The predicted arrival time at Earth from Run 1 was 21 January 2012T22:29Z and the predicated arrival time from Run 2 was 22 January 2012T00:52Z. By including the CME occurring on 18 January, Run 2 improves the forecasted arrival time by $2 \mathrm{~h} 23 \mathrm{~min}$. Figure 12 shows the simulated solar wind density/speed (top panel, with the density in black and speed in red) and the total magnetic field (bottom panel) from the two runs (Figures 12a and $12 b)$, with the arrival time being marked by the vertical lines. Note that because the two runs were performed during our real-time space weather operations, the time window for two runs is slightly different. The actual observed shock arrival time at ACE spacecraft is 22 January 2012T05:14Z as indicated by Figure 13. The top panel of Figure 13 shows the magnitude of IMF at ACE in black, the $z$ component of IMF in red, and the $y$ component of IMF in blue. The bottom panel of Figure 13 is the measured solar wind speed. The green vertical line indicates interplanetary shock arrival due to the CMEs.

[41] From the simulation results of the two runs, we can see that the earlier and slower CME on 18 January seems to merge together with the faster CME on 19 January and slow down its arrival by $2 \mathrm{~h}$ and $23 \mathrm{~min}$. Run 2 most likely reflects

Table 3. The Three CMEs Occurred in Quick Succession During 18-19 January 2012

\begin{tabular}{lcccr}
\hline CME Starting Time & Speed km/s & Direction LON/LAT in HEEQ & Half-Cone Angle (Degrees) & Time at 21.5 Rs \\
\hline 1. 2012-01 -18T 13:25Z & 520 & $3 /-24$ & 32 & $2012-01-18 T 20: 20 Z$ \\
2. 2012-01-19T10:12Z & 372 & $23 / 48$ & 69 & $2012-01-19 T 20: 51 Z$ \\
3. 2012-01-19T15:10Z & 1020 & $-21 / 46$ & $61-01-19 T 18: 34$ \\
\hline
\end{tabular}


(a) The run with 2 CMEs
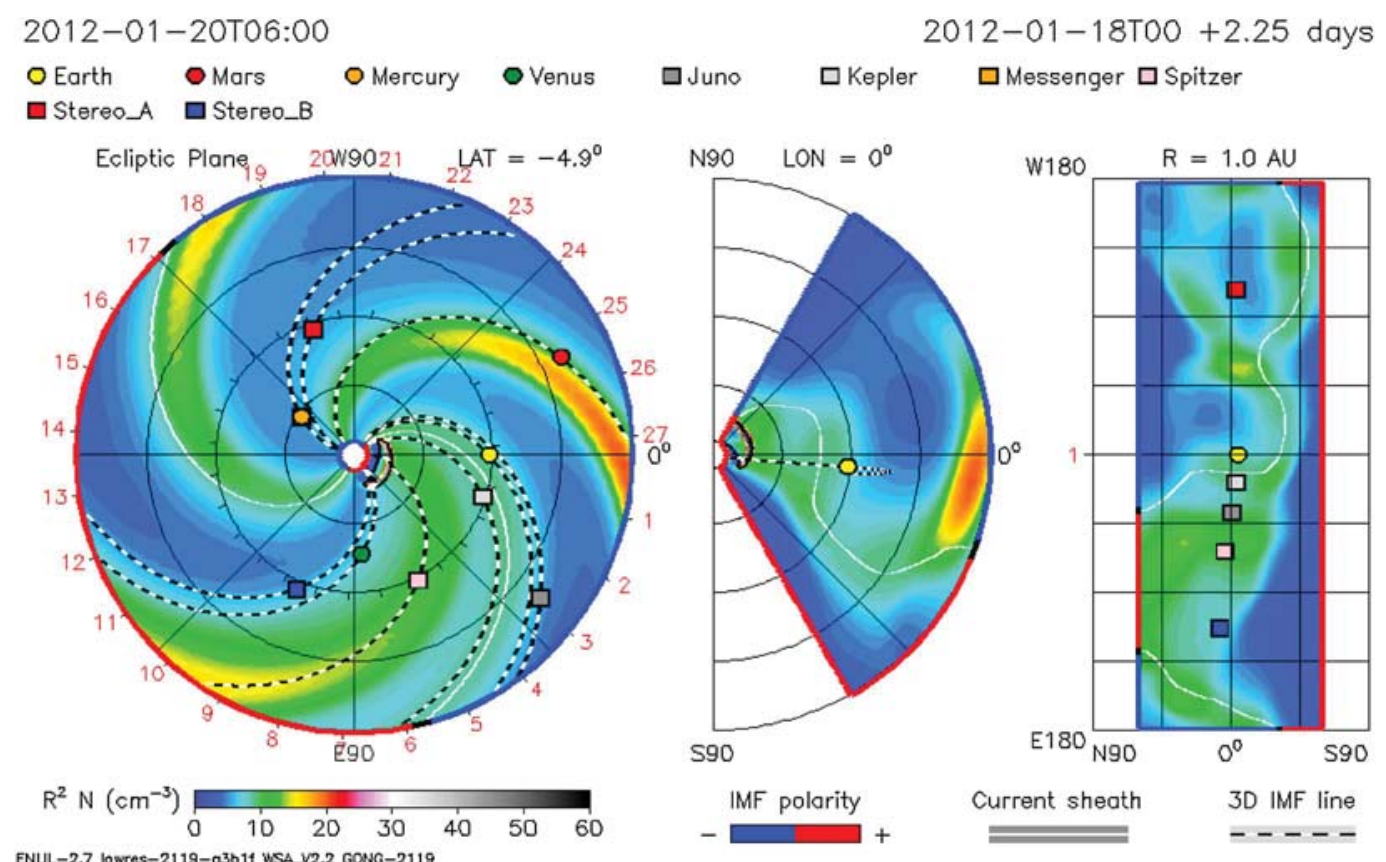

(b) The run with 3 CMEs
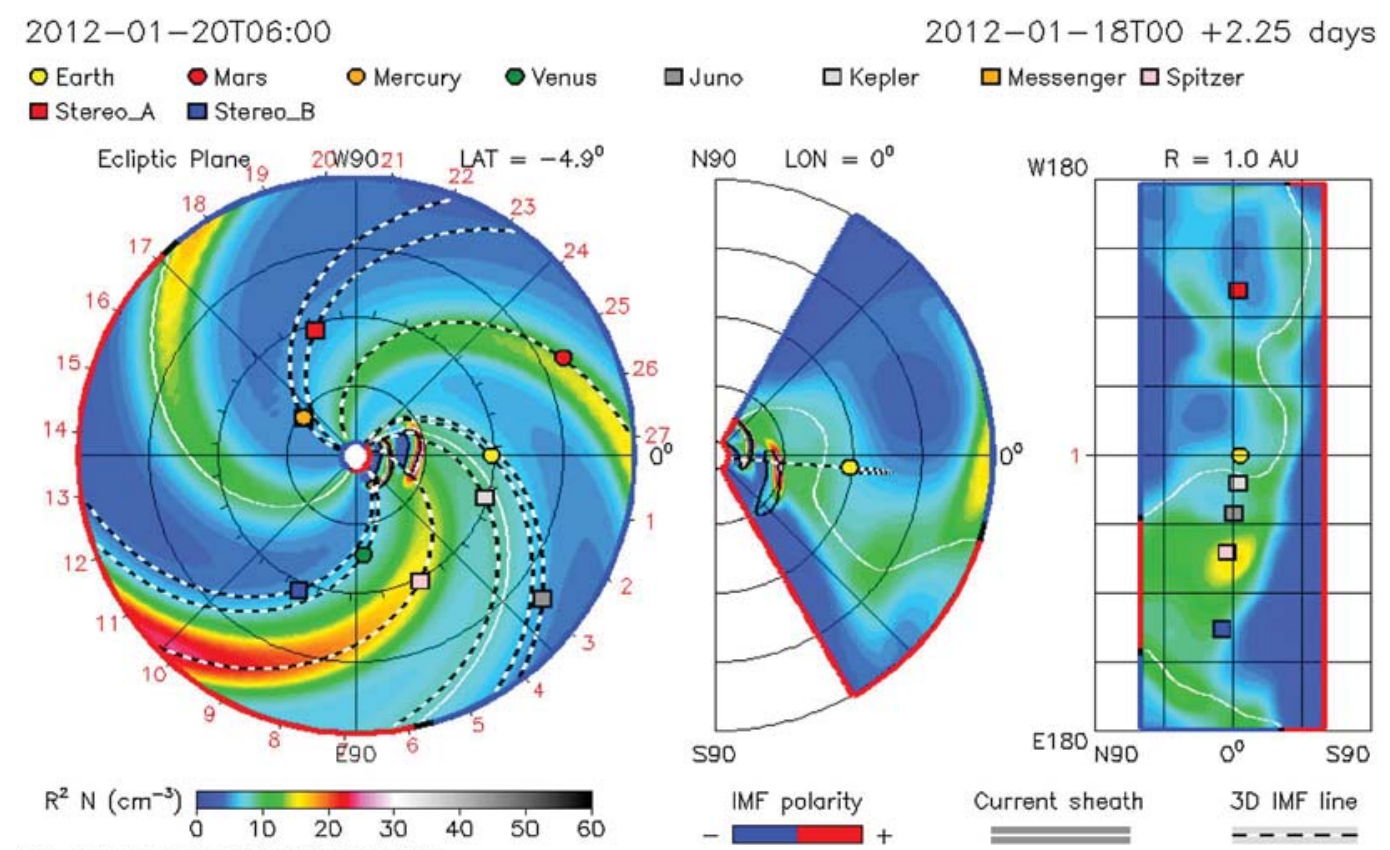

ENUL-2.7 lowres-2119-03b11 WSALV.2 GONG-2119

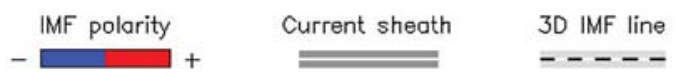

Figure 10. The simulated 3-D solar wind density map at 20 January 2012 06:00 UT for Run 1 (a) that includes two CMEs on 19 January 2012 and Run 2 (b) that includes CMEs in Run 1 and another CME on 18 January 2012. 
(a) The run with 2 CMEs
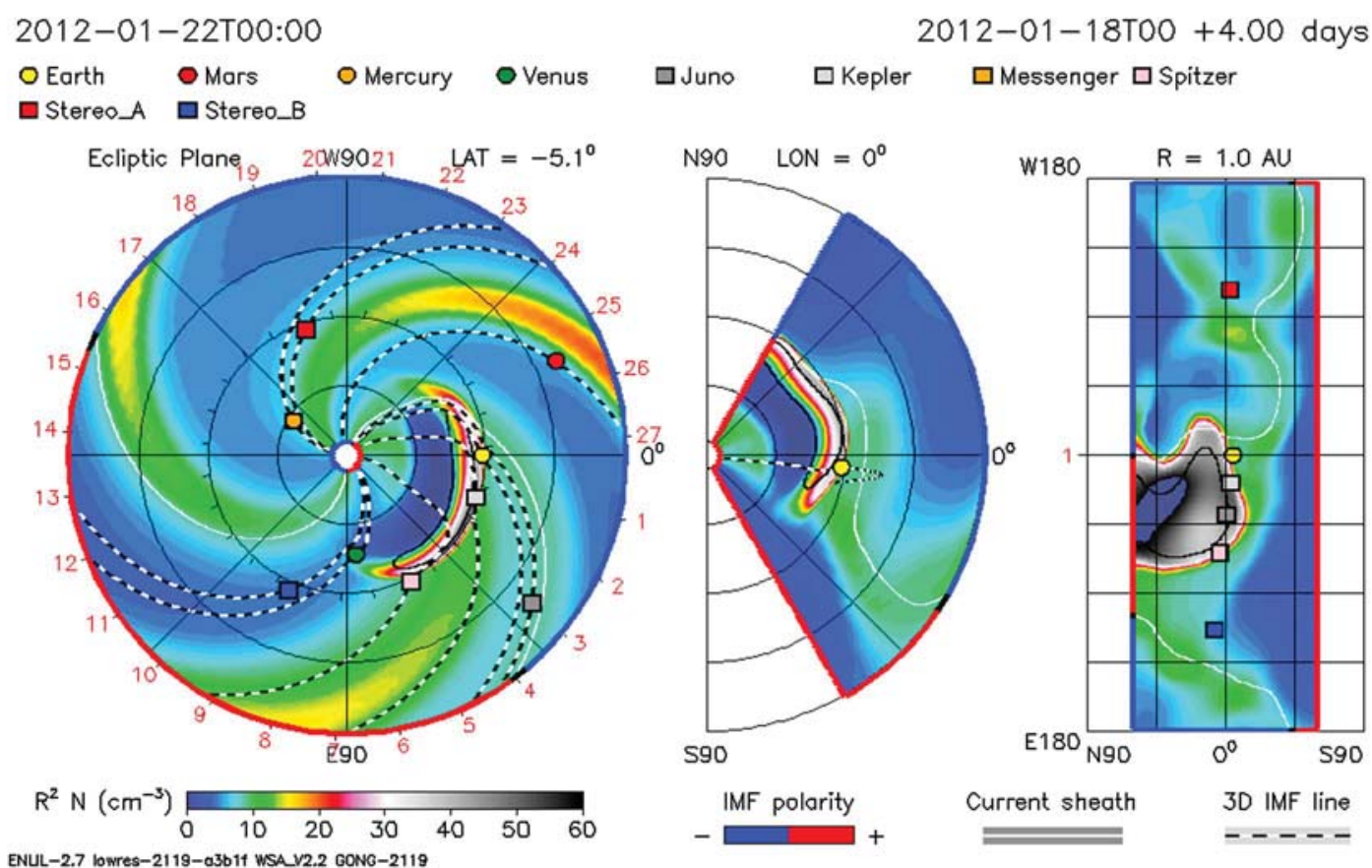

口Stereo_A 口Stereo_B
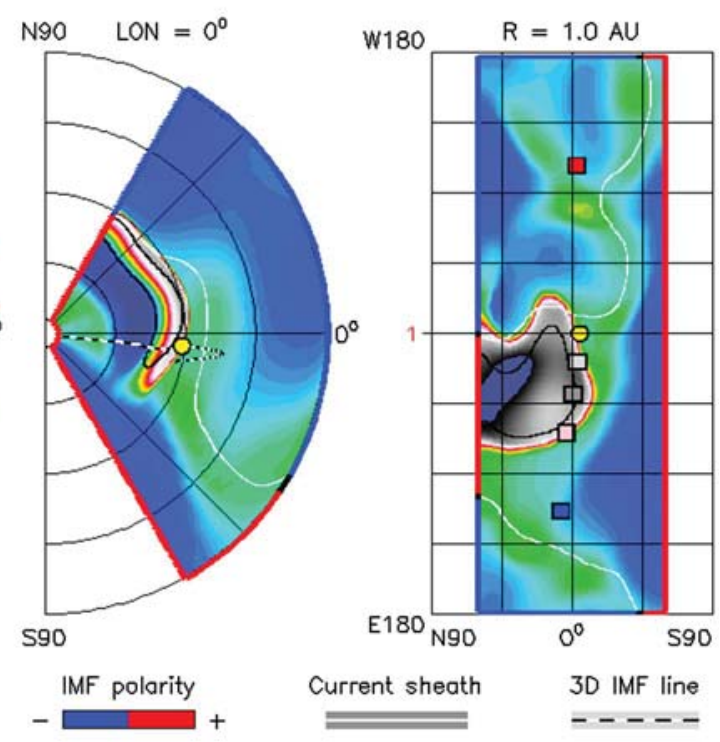

(b) The run with 3 CMES

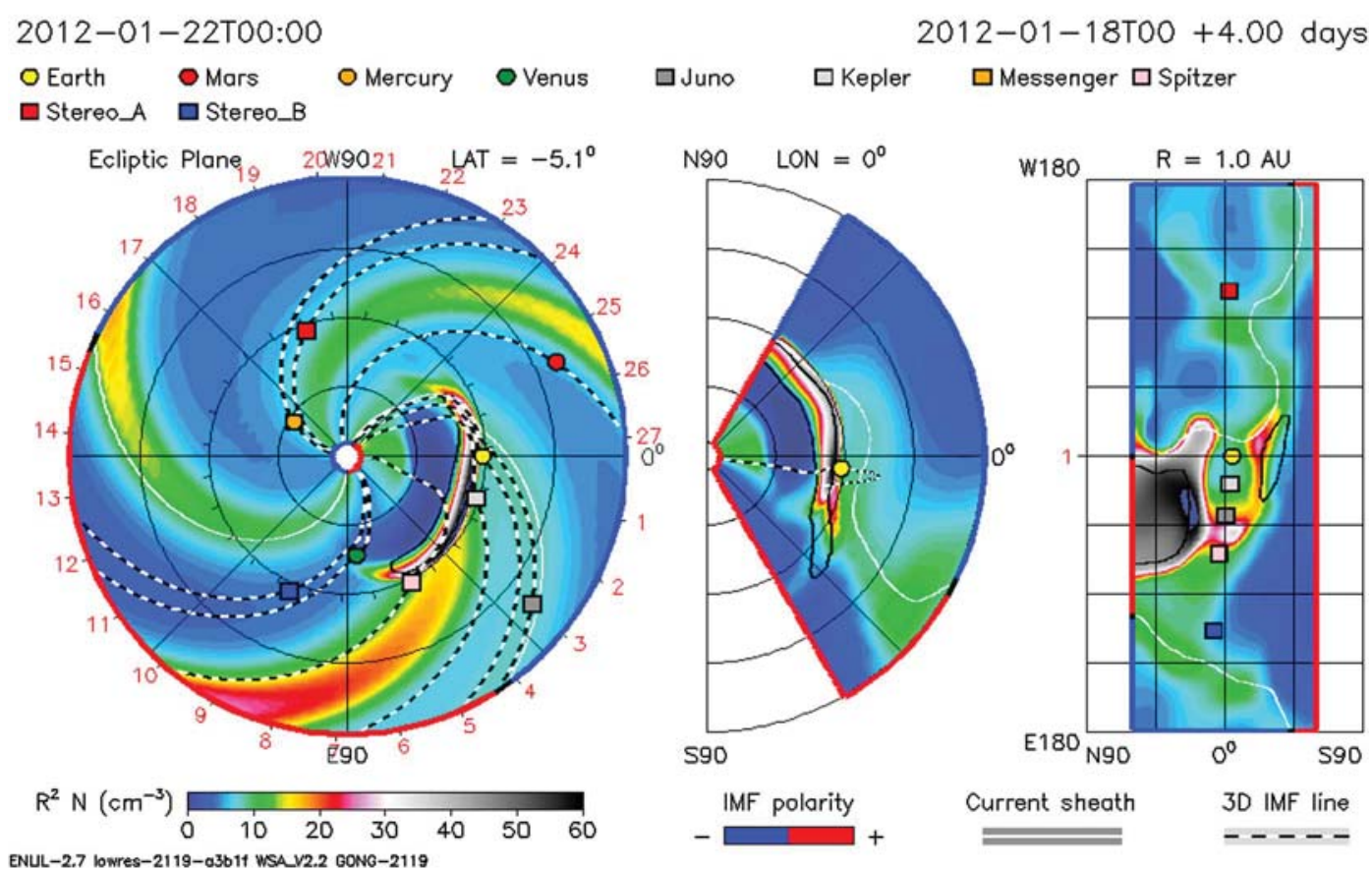

Figure 11. Similar to Figure 10, but showing the solar wind density map at 22 January 2012 00:00 UT for Run 1 and Run 2. 
(a) The run with 2 CMEs

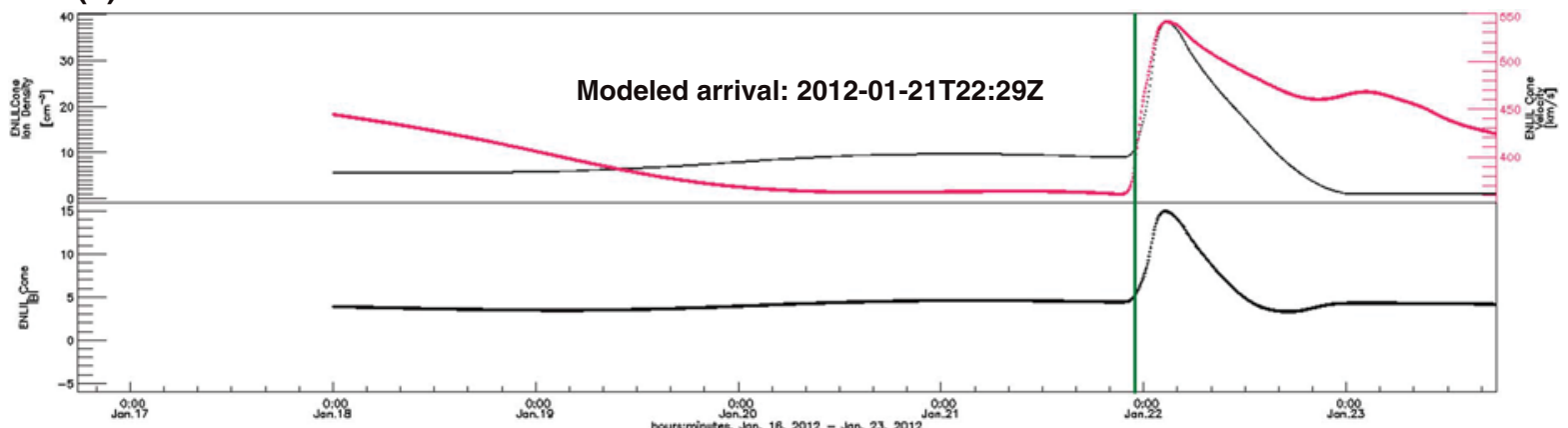

(b) The run with 3 CMEs

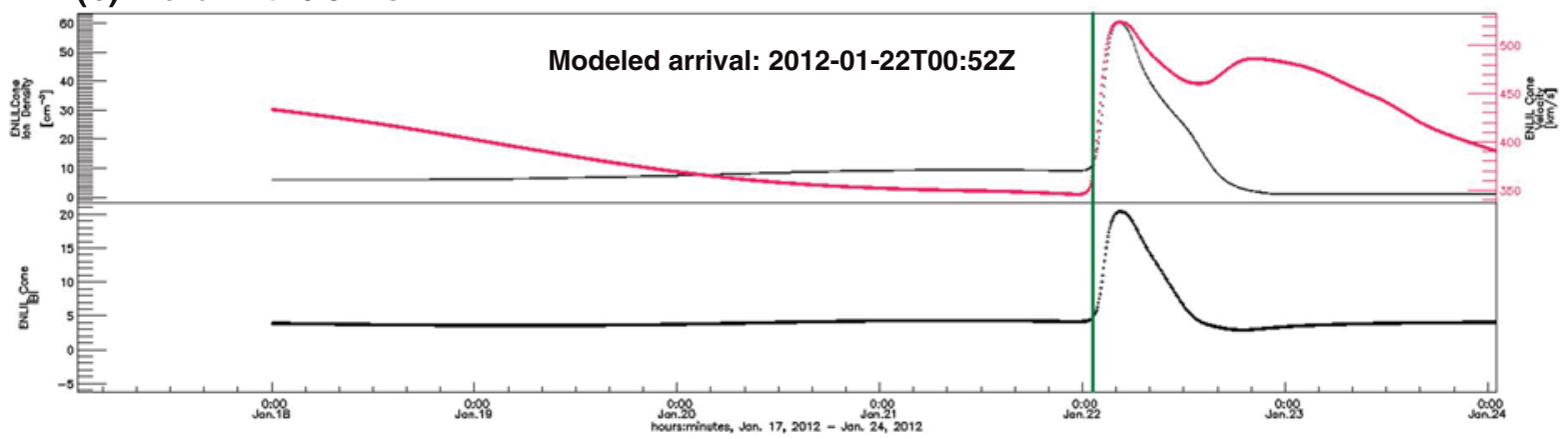

Figure 12. The simulated solar wind density/speed (top panel, with the density in black and speed in red) and the total magnetic field (bottom panel) from Run 1 and Run 2 (Figures 12a and 12b), with the arrival time being marked by the vertical lines. The predicted arrival time at Earth from Run 1 was 21 January 2012 22:29 UT and that from Run 2 was 22 January 2012 00:52 UT.

the actual ambient solar wind conditions more precisely. The interactions among CMEs with the ambient solar wind and other solar wind structures such as CIRs have been actively pursued as research topics [e.g., Gopalswamy et al., 2009]. Here we want to emphasize their importance in space weather operations, as indicated by this example (and many other examples not shown). As a general practice, all CMEs occur within a short period (roughly $24 \mathrm{~h}$ depending on individual situations) are included in the WSA+ENLIL+Cone simulation when assessing their arrival and impacts.

\subsection{The Importance of Other Parameters}

[42] In addition to those mentioned above, a number of other important factors must be taken into consideration.

[43] An accurate prediction of CME propagation and transport in interplanetary space requires an accurate portrait of CME parameters and the medium through which it travels. This paper's intent is not to dismiss previous research efforts in addressing these two essential aspects of the $\mathrm{CME}$ forecasting, but rather to reiterate their importance and to urge further efforts by the community to improve forecasting capability.

[44] Among a CME's kinetic attributes, its propagation direction has particular importance. It also tends to possess large uncertainties (for example, in comparison to speed and angular width) when determined operationally. The importance of CMEs' propagation direction to an object of impact in interplanetary space can be readily understood. Research results of some time ago [e.g., De Young and Hundhausen, 1973; D'Uston et al., 1981] demonstrate that CMEs are highly directional and that their effects at $1 \mathrm{AU}$ differ substantially depending on their propagation direction. However, the terminology of that era (everything was referred to as "flare" associated, and no distinction was made between flares and CMEs) limits their recognition as studies of CMEs. In addition, for people who are involved in operations, obtaining accurate information of the CME propagation direction can present practical concerns. Uncertainties in CME propagation direction are almost inevitable as data gaps in real-time coronagraph images are common and concurrent images from more than one vantage point are sometimes not possible. The CME direction not only determines how much an object of interest is affected and whether it is affected at all (flank impacts or a miss), but also determines what kind of ambient solar wind through which the CME propagates. So the influence of the propagation on the CME transport is evident. Simulation results of six WSA+ENLIL+Cone runs in Table 4 serve as a good example. Roughly based on the CME that occurred on 11 April 2013, an ensemble of runs was conducted to examine how the propagation affects its arrival time at Earth. The input parameters of the CME for each run are tabulated in Table 4 . For any two runs with the same speed $(1000 \mathrm{~km} / \mathrm{s}$, or $900 \mathrm{~km} / \mathrm{s}$, or $800 \mathrm{~km} / \mathrm{s}), 10^{\circ}$ 


\section{ZHENG ET AL.: FORECASTING CMES}

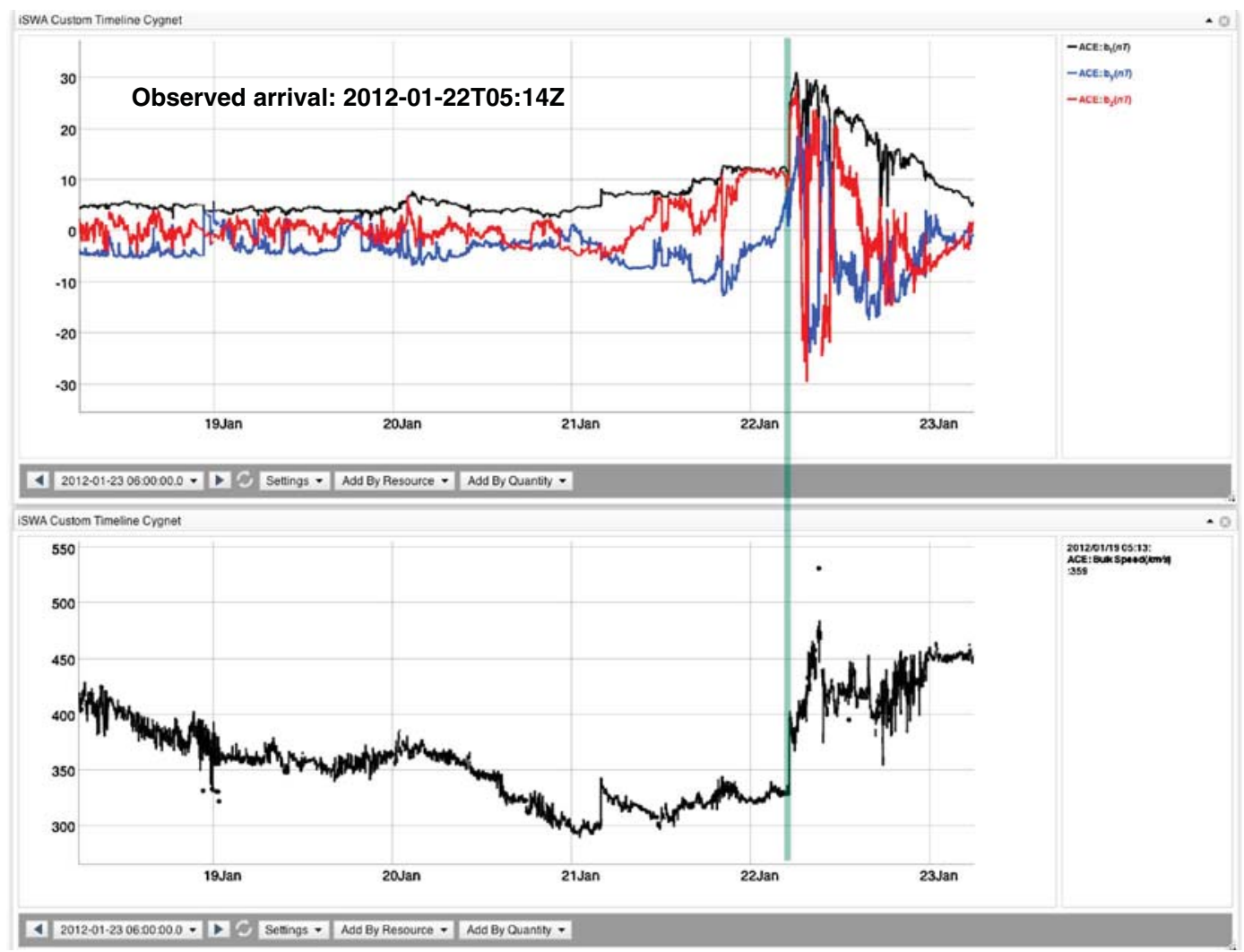

Figure 13. The top panel shows the magnitude of IMF at ACE in black, the $z$ component of IMF in red, and the $y$ component of IMF in blue. The bottom panel is the measured solar wind speed. The interplanetary shock arrival due to the CMEs (at 22 January 2012 05:14 UT) is indicated by the green vertical line.

longitudinal difference results in roughly $2 \mathrm{~h}$ difference in arrival time. It should be mentioned that the modeled $\mathrm{CME}$ for the comparative analysis is either $45^{\circ}$ or $35^{\circ}$ east off the Sun-Earth line. If the simulated CME has a more direct hit on Earth, the difference in arrival time could be bigger. Our results shown here serve as further confirmation of previous results [e.g., De Young and Hundhausen, 1973; D'Uston et al., 1981].

[45] Table 4 also indicates that CME speed is an important factor in determining its arrival time.

[46] Since CMEs have to propagate through the ambient solar wind, an accurate description of the ambient solar wind is also crucial. Even though this topic has been investigated extensively before [e.g., Case et al., 2008 and references therein], how to best represent the ambient solar wind in an operational setting remains a challenging task.

[47] A detailed investigation on how various CME parameters affect the arrival time will be left for a future study.

\section{Summary and Discussion}

[48] Better forecasting of a CME's propagation/evolution boils down to an improved representation of properties of the CME itself and the medium it travels through. Based on our operational experience, we have identified a few important considerations worthy of sharing, not only with regard to modeling CMEs, but also concerning the use of CME modeling results for additional benefits. Even though

Table 4. Effects of Propagation Direction on the Arrival Time of a CME

\begin{tabular}{lccrr}
\hline Time @ 21.5 Rs & Speed $(\mathrm{km} / \mathrm{s})$ & Direction LON/LAT in HEEQ & Half-Cone Angle (Degrees) & Arrival Time @ 1 AU \\
\hline 2013-04-11T10:50Z & 1000 & $-35 / 0$ & 55 & $2013-04-13 T 08: 19 Z$ \\
2013-04-11T10:50Z & 1000 & $-45 / 0$ & 55 & $2013-04-13 T 10: 12 Z$ \\
2013-04-11T10:58Z & 900 & $-35 / 0$ & 55 & $2013-04-13 T 11: 45 Z$ \\
2013-04-11T10:58Z & 900 & $-45 / 0$ & 55 & $2013-04-13 T 13: 37 Z$ \\
2013-04-11T11:03Z & 800 & $-35 / 0$ & 55 & $2013-04-13 T 15: 38 Z$ \\
2013-04-11T11:03Z & 800 & $-45 / 0$ & & $2013-04-13 T 17: 26 Z$ \\
\hline
\end{tabular}


existing observations relevant to CMEs are not ideal (and more is still needed), they are much better than before with the measurements made available from the recent launch of STEREO A, STEREO B, and SDO. Maximizing all the available information to get the best assessment of CME properties is emphasized. Besides the conventional use of WSA+ENLIL+Cone model results for CME arrival/impact forecasts, the simulation results can also provide a visual representation of the distribution of SEPs, which is stressed in the paper. The third important point is to include all CMEs occurring in quick succession where the time window for this guideline is set roughly as $24 \mathrm{~h}$. In addition, we have also identified a number of factors that affect CME arrival forecasting with a particular emphasis on the CME propagation direction.

[49] The coupled WSA+ENLIL+Cone model suite, as a sophisticated, physics-based 3-D model, is not only capable of performing tailored, complex calculations for research purpose, but also proves itself a powerful tool for space weather operations. Comparing to other global 3-D models, its fast running time (about 20 min each run) is another advantage. As mentioned above, however, it also has its own limitations. For example, a CME is modeled as an overpressured plasma cloud without its internal magnetic structure(s); shocks from CMEs are modeled to a limited degree.

[50] Real-time modeling and/or forecasting SEPs accurately continue to present one of the most challenging tasks in space weather research. While we should not ignore the added benefit of using the ICME modeling results to qualitatively assess where SEPs are expected in interplanetary space, caution needs to be exercised regarding its limitations. There are very uncertain relationships between shocks and SEPs as observed in many cases. The particle intensity and energy level of SEPs can vary greatly at shocks of nearly identical Mach number. The shock geometry, angle between B and the shock normal, and the required seed population of superthermal particles all play important roles in SEP acceleration [e.g., Reames, 1999, Tylka and Lee, 2006; Tylka et al., 2006; Li et al., 2009]. Shock strength alone cannot provide a very accurate prediction of SEPs. Thus, the MHD model shock strength only allows for a rough estimate of where SEPs might occur. If coupled with a particle transport code such as SEPMOD [Luhmann et al., 2007; Mewaldt et al., 2013], the extended WSA+ENLIL+Cone model could improve the SEP modeling capability. However, such SEP modeling results are not yet routinely available.

[51] To account for uncertainties in the initial CME parameters, an ensemble approach has been introduced and explored by our team members as a possible future direction [Pulkkinen et al., 2011]. It holds the promise of meaningfully characterizing the uncertainty involved in the CME modeling/forecasting process by providing a dynamic error bar. It should be noted, however, that accurate determination of the mean values of the CME parameters remains crucial in the ensemble approach. Until continuous operational data streams for CME parameter determination become a reality, scientific expertise within a forecasting center/team (and constant knowledge transfer from members who have the expertise to other team members) always needs to be invoked in forecasting CMEs.

[52] Acknowledgments. We thank all four reviewers for their time and constructive comments. We want to thank STEREO, SDO, SOHO, ACE, GOES teams, and others for the success of the mission and for making the data (particularly the real-time data) available. The authors would like to acknowledge the whole team of the Space Weather Research Center at NASA Goddard Space Flight Center and the Community Coordinated Modeling Center for their contribution. We thank Robert A. Herschbach for the editorial assistance. It should be mentioned that the majority of figures used in this paper were provided by iSWA (http://iswa.gsfc.nasa.gov).

\section{References}

Arge, C. N., and V. J. Pizzo (2000), Improvement in the prediction of solar wind conditions using near-real time solar magnetic field updates, J. Geophys. Res., 105(A5), 10,465-10,479.

Baker, D. N., et al. (2013), Solar wind forcing at Mercury: WSA-ENLIL model results, J. Geophys. Res. Space Physics, 118, 45-57, doi:10.1029/ 2012JA018064.

Boteler, D. H., R. J. Pirjola, and H. Nevanlinna (1998), The effects of geomagnetic disturbances on electrical systems at the earth's surface, Adv. Space Res., 22, 17-27.

Byrne, J. P., S. A. Maloney, R. T. James McAteer, J. M. Refojo, and P. T. Gallagher (2010), Propagation of an Earth-directed coronal mass ejection in three dimensions, Nat. Commun., 1, doi:10.1038/ncomms1077.

Cane, H. V. (2000), Coronal mass ejections and Forbush decreases, Space Sci. Rev., 93, 55-77.

Cane, H. V., and D. Lario (2006), An introduction to CMEs and energetic particles, Space Sci. Rev., 123, 45-56.

Cane, H. V., and I. G. Richardson (2003), Interplanetary coronal mass ejections in the near-Earth solar wind during 1996-2002, J. Geophys. Res., 108(A4), 1156, doi:10.1029/2002JA009817.

Cane, H. V., T. T. von Rosenvinge, C. M. S. Cohen, and R. A. Mewaldt (2003), Two components in major solar particle events, Geophys. Res. Lett., 30(12), 8017, doi:10.1029/2002GL016580.

Cane, H. V., R. A. Mewaldt, C. M. S. Cohen, and T. T. von Rosenvinge (2006), Role of flares and shocks in determining solar energetic particle abundances, J. Geophys. Res., 111, A06S90, doi:10.1029/ 2005JA011071.

Case, A. W., H. E. Spence, M. J. Owens, P. Riley, and D. Odstrcil (2008), Ambient solar wind's effect on ICME transit times, Geophys. Res. Lett., 35, L15105, doi:10.1029/2008GL034493.

Cliver, E. W. (2008), History of research on solar energetic particle events: The evolving paradigm, Proc. Int. Astron. Union, 4, 401-412, doi:10.1017/S1743921309029639.

Cliver, E. W., and A. G. Ling (2009), Astrophys. J., 690, 598.

Cohen, C. M. S., R. A. Mewaldt, R. A. Leske, A. C. Cummings, E. C. Stone, M. E. Wiedenbeck, E. R. Christian, and T. T. von Rosenvinge (1999), New observations of heavy-ion-rich solar particle events from ACE, Geophys. Res. Lett., 26(17), 2697-2700.

De Young, D. S., and A. J. Hundhausen (1973), Simulation of driven flare-associated disturbances in the solar wind, J. Geophys. Res., 78(19), 3633-3642, doi:10.1029/JA078i019p03633.

D'Uston, C., M. Dryer, S. M. Han, and S. T. Wu (1981), Spatial structure of flare-associated perturbations in the solar wind simulated by a two-dimensional numerical MHD model, J. Geophys. Res., 86(A2), 525-534, doi:10.1029/JA086iA02p00525.

Falkenberg, T. V., B. Vršnak, A. Taktakishvili, D. Odstrcil, P. MacNeice, and M. Hesse (2010), Investigations of the sensitivity of a coronal mass ejection model (ENLIL) to solar input parameters, Space Weather, 8, S06004, doi:10.1029/2009SW000555.

Falkenberg, T. V., S. Vennerstrom, D. A. Brain, G. Delory, and A. Taktakishvili (2011), Multipoint observations of coronal mass ejection and solar energetic particle events on Mars and Earth during November 2001, J. Geophys. Res., 116, A06104, doi:10.1029/ 2010JA016279. 


\section{ZHENG ET AL.: FORECASTING CMES}

Fry, C. D., W. Sun, C. S. Deehr, M. Dryer, Z. Smith, S.-I. Akasofu, M. Tokumaru, and M. Kojima (2001), Improvements to the HAF solar wind model for space weather predictions, J. Geophys. Res., 106(A10), 20,985-21,001, doi:10.1029/2000JA000220.

Gopalswamy, N. (2006), Properties of interplanetary mass ejections, Space Sci. Rev., 124, 145-168.

Gopalswamy, N., S. Yashiro, A. Lara, M. L. Kaiser, B. J. Thompson, P. T. Gallagher, and R. A. Howard (2003), Large solar energetic particle events of cycle 23: A global view, Geophys. Res. Lett., 30(12), 8015, doi:10.1029/2002GL016435.

Gopalswamy, N., S. Yashiro, G. Michalek, H. Xie, R. P. Lepping, and R. A. Howard (2005a), Solar source of the largest geomagnetic storm of cycle 23, Geophys. Res. Lett., 32, L12S09, doi:10.1029/2004GL021639.

Gopalswamy, N., L. Barbieri, G. Lu, S. P. Plunkett, and R. M. Skoug (2005b), Introduction to the special section: Violent Sun-Earth connection events of October-November 2003, Geophys. Res. Lett., 32, L03S01, doi:10.1029/2005GL022348.

Gopalswamy, N., B. Fleck, and J. B. Gurman (2005c), Major Scientific Results from SOHO on Coronal Mass Ejections, in Proceedings of Asia Pacific Regional Conference of IAA "Bring Space Benefits to the Asia Region", edited by M. Rao and R. Murphy, Astronautical Society of India, BANGALORE, INDIA.

Gopalswamy, N., P. Mäkelä, H. Xie, S. Akiyama, and S. Yashiro (2009), CME interactions with coronal holes and their interplanetary consequences, J. Geophys. Res., 114, A00A22, doi:10.1029/2008JA013686.

Guhathakurta, M. (2013), Interplanetary Space Weather: A new Paradigm, Eos Trans. AGU, 94(18), 165-172.

Hudson, H. S. (2011), Global Properties of Solar Flares, Space Sci. Rev., 158, 5, doi:10.1007/s11214-010-9721-4.

Kallenrode, M.-B. (2003), Current views on impulsive and gradual solar energetic particle events, J. Phys. G: Nucl. Part. Phys., 29, 965-981.

Lanzerotti, L. J. (2004), High-energy solar particles and human explorations, Space Weather, 3, S05002, doi:10.1029/2005SW000174.

Li, G., and G. P. Zank (2005), Mixed particle acceleration at CME-driven shocks and flares, Geophys. Res. Lett., 32, L02101, doi:10.1029/2004GL021250.

Li, G., G. P. Zank, O. Verkhoglyadova, R. A. Mewaldt, C. M. S. Cohen, G. M. Mason, and M. I. Desai (2009), Shock geometry and spectral breaks in large SEP events, Astrophys. J., 702, 998-1004.

Linker, J. A., and Z. Mikic (1995), Disruption of a helmet streamer by photospheric shear, Astrophys. J., 438, L45.

Liou, K., C.-C. Wu, M. Dryer, S.-T. Wu, D. B. Berdichevsky, S. Plunkett, R. A. Mewaldt, and G. M. Mason (2013), Magnetohydrodynamic fast shocks and their relation to solar energetic particle event intensities, Terr. Atmos. Ocean Sci., 24, 165-173, doi:10.3319/ TAO2012.05.08.01(SEC).

Luhmann, J. G., S. A. Ledvina, D. Krauss-Varban, D. Odstrcil, and P. Riley (2007), A heliospheric simulation-based approach to SEP source and transport modeling, Adv. Space Res., 40(3), 295-303.

Luhmann, J. G., S. A. Ledvina, D. Odstrcil, M. J. Owens, X.-P. Zhao, Y. Liu, and P. Riley (2010), Cone model-based SEP event calculations for applications to multipoint observations, Adv. Space Res., 46(1), $1-21$.

Mewaldt, R. A., C. M. S. Cohen, G. M. Mason, T. T. von Rosenvinge, R. A. Leske, J. G. Luhmann, D. Odstrcil, and A. Vourlidas (2013), Solar energetic particles and their variability from the sun and beyond, AIP Conf. Proc., 1539, 116-121, doi:10.1063/1.4811002.

Newell, P. T., T. Sotirelis, K. Liou, C.-I. Meng, and F. J. Rich (2007), A nearly universal solar wind-magnetosphere coupling function inferred from 10 magnetospheric state variables, J. Geophys. Res., 112, A01206, doi:10.1029/2006JA012015.

Odstrcil, D., and V. J. Pizzo (1999), Distortion of interplanetary magnetic field by three-dimensional propagation of CMEs in a structured solar wind, J. Geophys. Res., 104(A12), 28,225-28,239.

Odstrcil, D., J. A. Linker, R. Lionello, Z. Mikic, P. Riley, V. J. Pizzo, and J. G. Luhmann (2002), Merging of coronal and heliospheric two-dimensional MHD models, J. Geophys. Res., 107(A12), 1493, doi:10.1029/2002JA009334.

Odstrcil, D., V. J. Pizzo, J. A. Linker, P. Riley, R. Lionello, and Z. Mikic (2004a), Initial coupling of coronal and heliospheric numerical magnetohydrodynamic codes, J. Atmos. Sol. Terr. Phys., 66, 1311-1320.

Odstrcil, D., P. Riley, and X. P. Zhao (2004b), Numerical simulation of the 12 May 1997 interplanetary CME event, J. Geophys. Res., 109, A02116, doi:10.1029/2003JA010135.
Odstrcil, D., V. J. Pizzo, and C. N. Arge (2005), Propagation of the 12 May 1997 interplanetary coronal mass ejection in evolving solar wind structures, J. Geophys. Res., 110, A02106, doi:10.1029/ 2004JA010745.

Pirjola, R. (2007), Space weather effects on power grids, in Space weather-Physics and Effects, edited by V. Bothmer and I. Daglis, Springer, Berlin.

Pizzo, V., G. Millward, A. Parsons, D. Biesecker, S. Hill, and D. Odstrcil (2011), Wang-Sheeley-Arge-Enlil Cone Model Transitions to Operations, Space Weather, 9, S03004, doi:10.1029/2011SW000663.

Pulkkinen, A., A. Taktakishvili, D. Odstrcil, and P. MacNeice (2011), Ensemble forecasting of coronal mass ejection propagation in the interplanetary medium, poster presented at the NOAA Space Weather Workshop, Boulder, Colo., 23-26 April.

Pulkkinen, A., T. Oates, and A. Taktakishvili (2010), Automatic determination of the conic coronal mass ejection model parameters, Sol. Phys., 261, 115-126, doi:10.1007/s11207-009-9473-z.

Qiu, J., and V. B. Yurchyshyn (2005), Magnetic reconnection flux and coronal mass ejection velocity, Astrophys. J., 634, L121.

Qiu, J., H. Wang, C. Z. Cheng, and D. E. Gary (2004), Magnetic Reconnection and Mass Acceleration in Flare-Coronal Mass Ejection Events, Astrophys. J., 604, 900.

Reames, D. V. (1999), Particle acceleration at the Sun and in the heliosphere, Space Sci. Rev., 90, 413-491.

Riley, P., J. A. Linker, Z. Mikic', D. Odstrcil, T. H. Zurbuchen, D. Lario, and R. P. Lepping (2003), Using an MHD simulation to interpret the global context of a coronal mass ejection observed by two spacecraft, J. Geophys. Res., 108(A7), 1272, doi:10.1029/2002JA009760.

Schatten, K. H. (1971), Current sheet magnetic model for the solar corona, Cosmic Electrodyn., 2, 232-245.

Shen, F., X. S. Feng, S. T. Wu, C. Q. Xiang, and W. B. Song (2011), Three-dimensional MHD simulation of the evolution of the April 2000 CME event and its induced shocks using a magnetized plasma blob model, J. Geophys. Res., 116, A04102, doi:10.1029/2010JA015809.

Taktakishvili A., M. Kuznetsova, P. MacNeice, M. Hesse, L. Rastätter, A. Pulkkinen, A. Chulaki, and D. Odstrcil (2009), Validation of the coronal mass ejection predictions at the Earth orbit estimated by ENLIL heliosphere cone model, Space Weather, 7, S03004, doi:10.1029/2008SW000448.

Taktakishvili, A., A. Pulkkinen, P. MacNeice, M. Kuznetsova, M. Hesse, and D. Odstrcil (2011), Modeling of coronal mass ejections that caused particularly large geomagnetic storms using ENLIL heliosphere cone model, Space Weather, 9, S06002, doi:10.1029/ 2010 SW000642.

Tóth, G., et al. (2005), Space Weather Modeling Framework: A new tool for the space science community, J. Geophys. Res., 110, A12226, doi:10.1029/2005JA011126.

Tylka, A. J., and M. A. Lee (2006), A model for spectral and compositional variability at high energies in large, gradual solar particle events, Astrophys. J., 646, 1319.

Tylka, A. J., C. M.S. Cohen, W. F. Dietrich, M. A. Lee, C. G. Maclennan, R. A. Mewaldt, C. K. Ng, and D. V. Reames (2006), A comparative study of ion characteristics in the large gradual solar energetic particle events of 2002 April 21 and 2002 August 24, Astrophys. J. Suppl. Ser., 164, 536

Vršnak, B., T. Žic, T. V. Falkenberg, C. Möstl, S. Vennerstrom, and D. Vrbanec (2010), The role of aerodynamic drag in propagation of interplanetary coronal mass ejections, Astron. Astrophys., 512, A43, doi:10.1051/0004-6361/200913482.

Wang, Y. M., and N. R. Sheeley Jr. (1995), Solar implications of Ulysses interplanetary field measurements, Astrophys. J., 447, L143-L146.

Wang, Y., and J. Zhang (2007), A comparative study between eruptive $X$-class flares associated with coronal mass ejections and confined X-class flares, Astrophys. J., 665, 1428.

Webb, D. F., and T. A. Howard (2012), Coronal Mass Ejections: Observations, Living Rev. Solar Phys., 9, 3, lrsp-2012-3. URL (accessed 17 April 2013), http://www.livingreviews.org/lrsp-2012-3.

Woods, T. N., et al. (2010), Extreme Ultraviolet Variability Experiment (EVE) on the Solar Dynamics Observatory (SDO): Overview of Science Objectives, Instrument Design, Data Products, and Model Developments, Sol. Phys., 275, 115-143, doi:10.1007/s11207-0099487-6.

Wu, S. T., M. Dryer, and S. M. Han (1983), Non-planar MHD model for solar flare-generated disturbances in the heliospheric equatorial plane, Sol. Phys., 84, 395-418. 


\section{ZHENG ET AL.: FORECASTING CMES}

Wu, C.-C., M. Dryer, S. T. Wu, B. E. Wood, C. D. Fry, K. Liou, and S. Plunkett (2011), Global three-dimensional simulation of the interplanetary evolution of the observed geoeffective coronal mass ejection during the epoch 1-4 August 2010, J. Geophys. Res., 116, A12103, doi:10.1029/2011JA016947.

Xie, H., L. Ofman, and G. Lawrence (2004), Cone model for halo CMEs: Applications to space weather forecasting, J. Geophys. Res., 109, A03109, doi:10.1029/2003JA010226.

Yashiro, S., N. Gopalswamy, S. Akiyama, G. Michalek, and R. A. Howard (2005), Visibility of coronal mass ejections as a function of flare location and intensity, J. Geophys. Res., 110, A12S05, doi:10.1029/2005JA011151.

Zank, G. P., G. Li, and O. P. Verkhoglyadova (2007), Particle acceleration at interplanetary shocks, Space Sci. Rev., 130, 255, doi:10.1007/ s11214-007-9214-2.
Zhang, J., K. P. Dere, R. A. Howard, M. R. Kundu, and S. M. White (2001), On the temporal relationship between coronal mass ejections and flares, Astrophys. J., 559, 452.

Zhang, J., K. P. Dere, R. A. Howard, and A. Vourlidas (2004), A study of the kinematic evolution of coronal mass ejections, Astrophys. J., 604, 420.

Zhao, X. P., S. P. Plunkett, and W. Liu (2002), Determination of geometrical and kinematical properties of halo coronal mass ejections using the cone model, J. Geophys. Res., 107(A8), 1223, doi:10.1029/ 2001JA009143.

Zhou, Y. F., X. S. Feng, S. T. Wu, D. Du, F. Shen, and C. Q. Xiang (2012), Using a 3-D spherical plasmoid to interpret the Sun-to-Earth propagation of the 4 November 1997 coronal mass ejection event, J. Geophys. Res., 117, A01102, doi:10.1029/2010JA016380. 